\title{
Health Literacy and Family Factors in the Transition to Adult Care in Adolescents with Type I Diabetes
}

\author{
Ellen M. Manegold \\ West Virginia University, emmanegold@mix.wvu.edu
}

Follow this and additional works at: https://researchrepository.wvu.edu/etd

Part of the Child Psychology Commons, and the Health Psychology Commons

\section{Recommended Citation \\ Manegold, Ellen M., "Health Literacy and Family Factors in the Transition to Adult Care in Adolescents with Type I Diabetes" (2019). Graduate Theses, Dissertations, and Problem Reports. 4063. \\ https://researchrepository.wvu.edu/etd/4063 \\ This Dissertation is protected by copyright and/or related rights. It has been brought to you by the The Research Repository @ WVU with permission from the rights-holder(s). You are free to use this Dissertation in any way that is permitted by the copyright and related rights legislation that applies to your use. For other uses you must obtain permission from the rights-holder(s) directly, unless additional rights are indicated by a Creative Commons license in the record and/ or on the work itself. This Dissertation has been accepted for inclusion in WVU Graduate Theses, Dissertations, and Problem Reports collection by an authorized administrator of The Research Repository @ WVU. For more information, please contact researchrepository@mail.wvu.edu.}


Health Literacy and Family Factors in the Transition to Adult Care in Adolescents with Type 1 Diabetes

Ellen M. Manegold, M.S.

Dissertation submitted to the Eberly College of Arts and Sciences at West Virginia University

in partial fulfillment of the requirements for the degree of

Doctor of Philosophy in

Psychology

Christina L. Duncan, Ph.D., Chair

Elisa Krackow, Ph.D.

Julie Hicks Patrick, Ph.D.

Pamela Murray, M.D., M.H.P.

Department of Psychology

\section{Morgantown, West Virginia}

2019

Keywords: Type 1 Diabetes, Transition, Health Literacy, Adolescents, Family, Glycemic Control Copyright 2019, Ellen M. Manegold 


\begin{abstract}
Health Literacy and Family Factors in the Transition to Adult Care in Adolescents with Type I

Diabetes

Ellen M. Manegold

Successful management of type 1 diabetes (T1DM) in adolescence involves multiple daily tasks, developmental changes, and the expectation of transition to an adult provider. Health literacy is one variable to consider in the context of transition, as studies have demonstrated the correlation of parental health literacy with health behaviors and outcomes, yet not studied adolescent health literacy in T1DM. Family factors (e.g., management responsibility, diabetes-specific family conflict, parental support) also are important to behavior and health outcomes in adolescents with T1DM. Study aims were to: 1) examine the association of adolescent health literacy to transition readiness and health outcome in T1DM; and 2) explore the extent to which family factors serve as moderators in health literacy predicting transition readiness and health outcome. Sixty-five youth and their caregivers completed measures. Results indicated a significant positive correlation among health literacy and T1DM knowledge. Higher parental responsibility was significantly correlated with greater written health literacy, greater transition readiness, and fewer parental supportive behaviors. Higher written health literacy was associated with lower family conflict. The relations among health literacy and transition readiness and glycemic control were not significant. No family factors were found to be moderators for health literacy and transition readiness or glycemic control. Given the complex definition of health literacy and dearth of comprehensive validated measures in adolescents, our measures may not have adequately reflected global health literacy in the context of disease management. Future directions include studying additional aspects of health literacy and other variables potentially impacting health behaviors.
\end{abstract}




\section{Acknowledgements}

My time at West Virginia University has afforded me the opportunity to work with numerous individuals whose work I admire. Training with my mentor, Christina Duncan, Ph.D., has been a tremendous gift, as she has guided me from day one in learning important professional and personal lessons. I am incredibly grateful for past and present members of the Pediatric Lab for Adherence and Transition who were instrumental in many ways throughout this process. I am indebted to the families at the WVU Medicine Pediatric Endocrinology Clinic for their participation and trust in our research team. Similarly, this project could not have been completed without the support of the medical team, including Brian Ely, MD, Evan Jones, MD, Leah Woodburn, MS, RD, LD, and Daphne Barlow, CDE. Thank you to my committee members, Elisa Krackow, Ph.D., Julie Hicks Patrick, Ph.D., and Pamela Murray, MD, MHP, for their time and expertise on this project.

Personally, I am grateful to my parents, Ed and Mary Lou Manegold, for their unwavering support during these past five years. I appreciate the encouragement of my family members and friends, including Jon Stoltman and the StoltFamily, who were invested in this journey. A special thank you to those who called, wrote, and even traveled to visit the mountains and swamp. I share this achievement with all of you. 
Table of Contents

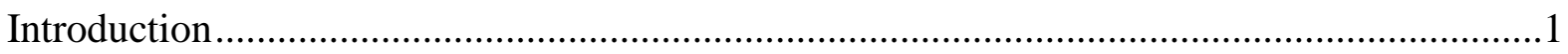

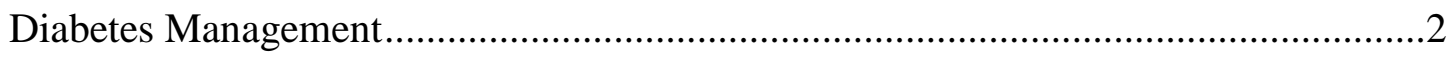

Transition to Adult Care .......................................................................................

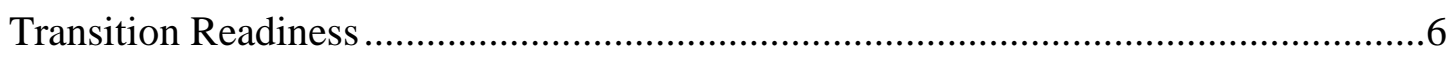

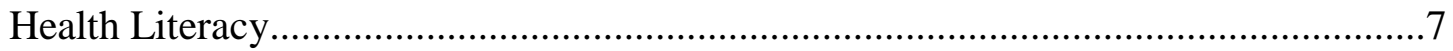

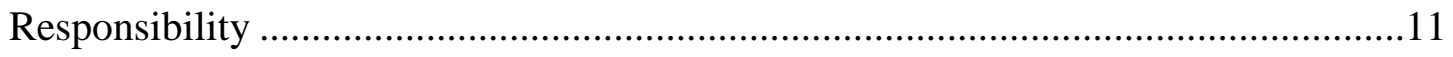

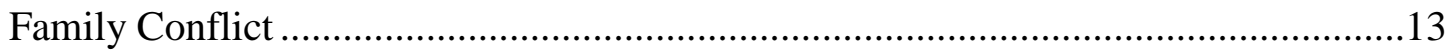

Supportive Parental Behaviors..................................................................... 16

Summary and Rationale for Current Study .................................................. 17

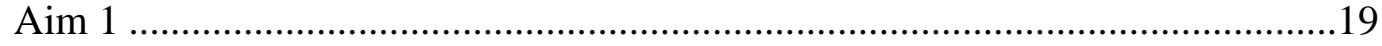

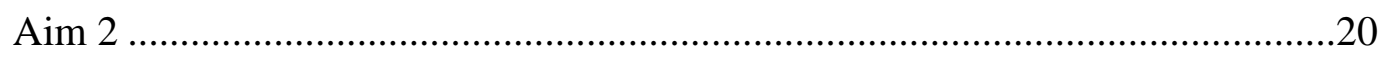

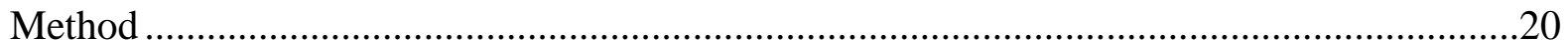

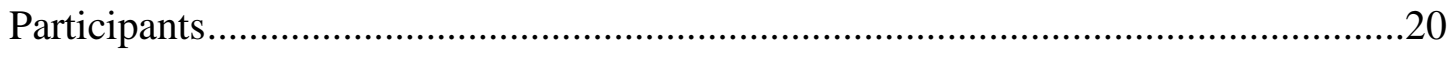

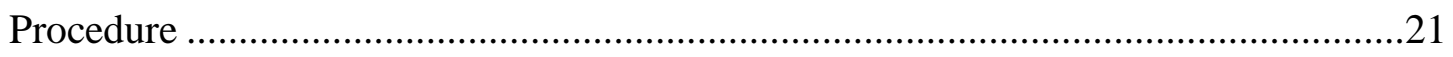

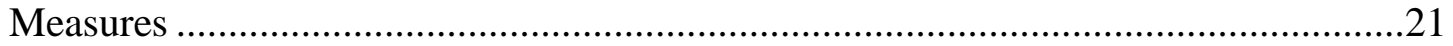

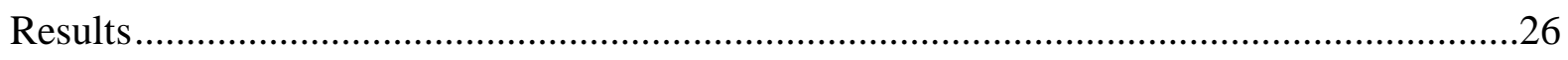

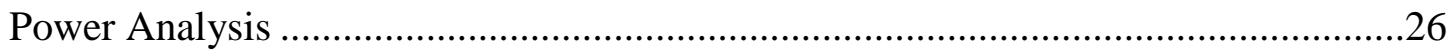

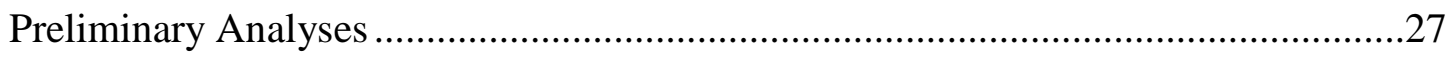

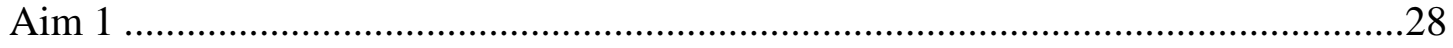

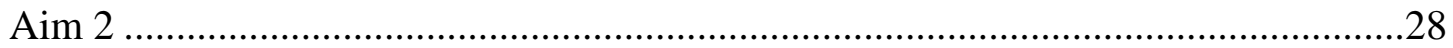

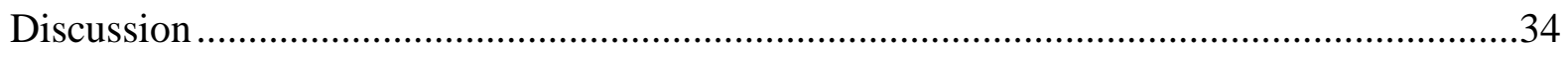

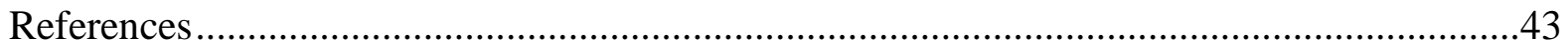




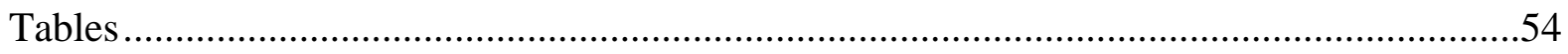

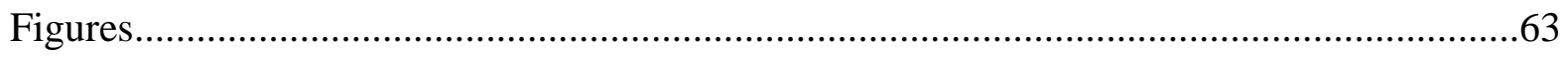

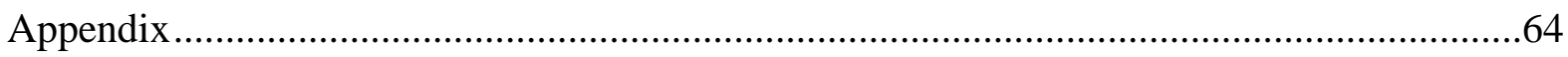




\section{List of Tables}

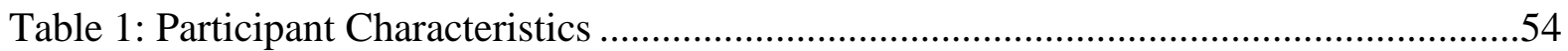

Table 2: Primary Variable Raw Score Descriptives .....................................................56

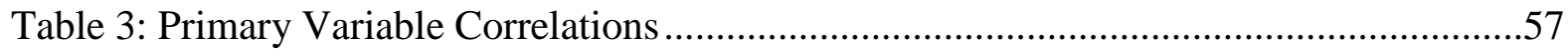

Table 4: Demographic Variable Correlations with Primary Variables ..................................58

Table 5: Moderation Effect of Family Factors on the Relation between Written Health

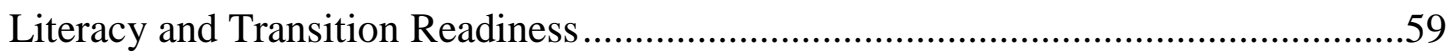

Table 6: Moderation Effect of Family Factors on the Relation between Written Health

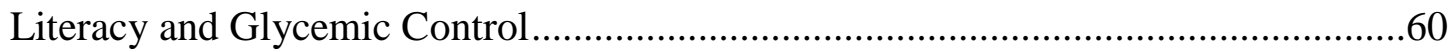

Table 7: Moderation Effect of Family Factors on the Relation between Numeric Health

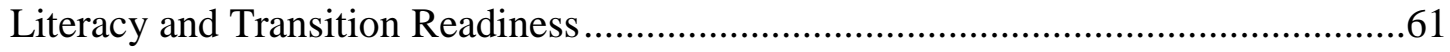

Table 8: Moderation Effect of Family Factors on the Relation between Numeric Health

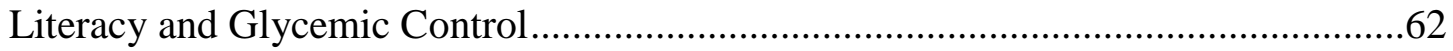




\section{List of Figures}

Figure 1: Proposed moderation model of family factors as moderators in adolescent health literacy predicting transition readiness and health outcome .63 
Health Literacy and Family Factors in the Transition to Adult Care in Adolescents with Type 1

Diabetes

Introduction

Type 1 diabetes (T1DM; formerly known as juvenile-onset diabetes or insulin-dependent diabetes mellitus) is an autoimmune disease that affects the body's production of insulin in the pancreas (Centers for Disease Control and Prevention [CDC], 2017). Insulin is a hormone crucial for obtaining energy from food and lowering blood glucose levels (CDC, 2017; Juvenile Diabetes Research Foundation [JDRF], 2019). In individuals with T1DM, the body's immune system attacks and destroys beta cells, which are responsible for creating insulin (CDC, 2017; JDRF, 2017). As a result, the body has a decrease in or lack of insulin production and secretion (CDC, 2017). Symptoms may include extreme thirst, frequent urination, drowsiness, increased appetite, abrupt weight loss, sudden changes in vision, and difficulty breathing (JDRF, 2019). Long-term, T1DM can result in kidney failure, loss of vision, damage to nerves, cardiac complication, and issues during pregnancy (JDRF, 2019). Livingstone and colleagues (2015) estimated that those living with T1DM have a shorter life expectancy. On average, females with T1DM will live almost 13 years shorter, and males will live over 11 years shorter compared with a general Scottish population. Although the exact cause of T1DM is not yet known, it is speculated that genetic and environmental components are involved in disease development (JDRF, 2019).

As its previous names suggest, T1DM is typically diagnosed in childhood or adolescence, although it can be diagnosed at any age (Dabelea et al., 2014; JDRF, 2019). It is estimated that about 200,000 youth ( 1 in 433) are living with T1DM in the United States, with approximately 1.25 million total cases across all ages (Dabelea et al., 2014; JDRF, 2019; Pettitt, et al., 2014). 
The most recent prevalence study estimated that almost 20,000 youth experience T1DM onset per year (CDC, 2014). Non-Hispanic, white youth account for the highest number of new T1DM diagnoses (CDC, 2014). T1DM rates in youth under 20 years old are expected to grow significantly. After adjusting for demographic variables, the incidence of T1DM increased 1.8\% per year from 2002 to 2012 (Mayer-Davis et al., 2017). Furthermore, it is estimated that about 5 million people in the United States will have T1DM by 2050, with almost 600,000 of them being youth (Dabelea et al., 2014; Impreatore et al., 2012).

\section{Diabetes Management}

Although research is currently being conducted, there is no known prevention or cure for T1DM (CDC, 2017; JDRF, 2019). Treatment for T1DM requires injections or a pump to deliver insulin to the body while also accounting for eating and other activities (JDRF, 2019). This requires multiple mathematical calculations to determine how much insulin to deliver over the course of a day to maintain appropriate blood glucose levels, account for foods consumed, or correct abnormal levels (American Diabetes Association [ADA], 2019b). There are over 20 different forms of insulin which have different onsets, peak times, durations, and strengths (ADA, 2019b). All of these factors account for which type, the timing, and how much insulin is needed. To complicate insulin injections further, placement also affects blood glucose levels; therefore, it is necessary to maintain the same general injection areas on the body at different times (e.g., before dinner in a similar area), yet rotate to different sites within the areas to maintain insulin reliability (ADA, 2019b). Lack of site rotation may result in lipodystrophychanges in fat under the skin that may affect insulin absorption (Kadiyala, Walton, \& Sathyapalan, 2014). 
Although many providers begin their patients on injections that distribute insulin to the muscle, some patients may be eligible to use a pump to maintain blood glucose control (ADA, 2019b). Pumps are small electronic devices that look somewhat like a pager that deliver insulin through a catheter for continuous and patient-directed doses (ADA, 2019b). An endocrinologist may recommend a pump if the patient has demonstrated good blood glucose control using injections and a pump would be amenable to their lifestyle (ADA, 2019b). As a result, patients who use pumps may no longer have to administer insulin injections, resulting in easier, more accurate insulin administration and increased lifestyle flexibility (ADA, 2019b). However, it is noteworthy that patients with pumps continue to need to monitor their food consumption and activity level, making calculations so that they can adjust insulin delivery through their pump, though these adjustments are typically less frequent. Some disadvantages of pump use include extensive education (up to one day training), health complications if the catheter is not appropriately placed, expense if not covered by insurance, and the constant wearing of a device attached to the body (ADA, 2019b). Overall, regardless of the insulin administration method, maintaining appropriate blood glucose levels is a complex routine.

Although insulin administration is a major component of T1DM self-management, there are other considerations that make this an involved condition to manage. To maintain appropriate blood glucose levels, people with T1DM should count the amount of carbohydrates that they eat for each meal or snack (ADA, 2019a). Patients must be aware of their starting blood sugar and amount of carbohydrates consumed in order to calculate what insulin is necessary (ADA, 2019a). Another important component of T1DM management is physical activity. Consistent exercise has been shown to improve blood glucose levels long-term, yet it can result in short-term changes (ADA, 2019a). Blood glucose may increase or decrease during and after physical 
activity depending on the starting level, as well as activity intensity and duration, thereby requiring close monitoring (ADA, 2019a). Consequently, lifestyle behaviors combined with repeated calculations for insulin administration make T1DM a burdensome condition.

Proper glycemic control is one of the major aims of T1DM management. Good glycemic control is associated with delayed onset and slowed progression of T1DM complications such as vision loss or impairment (retinopathy), kidney damage potentially leading to kidney failure (nephropathy), and nerve damage (neuropathy; The Diabetes Control and Complications Trial Research Group, 1993). Blood glucose levels must be monitored multiple times per day (e.g., before meals, before participation in physical activity, before bedtime) to determine how much insulin is necessary (JDRF, 2019; ADA, 2019b). These levels are obtained when a drop of blood, usually from the fingertip, is positioned on a blood glucose meter strip and read by an electronic meter device (JDRF, 2019). These meters provide prompt feedback and store the event to track monitoring over time (JDRF, 2019). More recently, continuous glucose monitoring devices can be attached to the body and give current readings, though still require some finger pricks throughout the day (JDRF, 2019). The ADA (2019) further recommends that glycated hemoglobin (HbA1c) levels are monitored at clinic appointments as a global measure of glycemic control over the past two to three months (Jeffcoate, 2004). In the general pediatric population, HbA1c levels less than 7.5\% are recommended (ADA, 2019).

The overarching goal in diabetes management is to maintain healthy blood glucose levels (JDRF, 2019). Unfortunately, even those who adhere to their prescribed regimen may still be at risk for the long-term complications noted earlier (JDRF, 2019). All of this treatment comes at a significant cost, as well. Annual healthcare costs associated with T1DM are estimated to be \$14 billion in the United States alone (JDRF, 2019). Given the significant health and monetary costs, 
increasing prevalence in youth, and burdensome daily treatment regimen, youth with T1DM are an important population to study in order to improve behavioral and health outcomes.

\section{Transition to Adult Care}

Transition from pediatric to adult care is a crucial time point to consider in successful disease self-management for those living with chronic illnesses. During this time period, most patients experience multiple crucial developmental changes (Arnett, 2000). For instance, emerging adults engage in new responsibilities traditionally held by caregivers, including those with implications for medical care (e.g., legal decision-making status). In addition, identity exploration is a hallmark of this developmental period. This includes changes across a variety of domains, including vocation, romantic relationships, and worldview.

For patients with T1DM, it can be a time full of additional challenges, particularly given the high complexity of its treatment regimen. Moreover, glycemic control tends to become worse during adolescence due to a variety of potential factors, including changing hormones, decreased adherence to insulin treatment, and inconsistent clinic attendance (Ball et al., 2006; Jacobson et al., 1997; Morris et al., 1997). This poor glycemic control tends to peak in late adolescence and improve in early adulthood (Bryden et al., 2001; Insabella, Grey, Knafl, \& Tamborlane, 2007). As stated previously, poor disease management measured by glycemic control places adolescents at an increased risk for T1DM-related complications, including hypertension, retinopathy, cognitive impairment, and death (Bryden et al., 2001). In one longitudinal study, $30 \%$ of adolescents with T1DM experienced serious complications, demonstrating that this is a vital developmental time period for T1DM management (Bryden et al., 2001). 


\section{Transition Readiness}

To successfully complete the active transition process from pediatric to adult care, patients must be able to complete tasks related to disease management, decision-making, and self-advocacy (Sawicki et al., 2011). According to an extension of the Social-Ecological Model of Adolescents and Young Adult Readiness for Transition (SMART), transition readiness is a complicated construct, with multiple variables interacting to influence transition readiness and outcomes in pediatric populations (Pierce \& Wysocki, 2015). Preexisting variables (e.g., demographics, culture) influence modifiable factors (e.g., skills, beliefs/expectations, relationships/communication), which then affect transition readiness (Pierce \& Wysocki, 2015). Patient transition readiness impacts transition outcomes (i.e., behavioral, biomedical, emotional), and both transition readiness and outcomes are influenced by systemic factors (e.g., social support, health care; Pierce \& Wysocki, 2015).

Due to the complexity of the construct, transition readiness can be difficult to assess. Transition readiness is frequently measured by evaluating a patient's reported ability to perform specific actions deemed important to self-management, though many of these measures' psychometric properties have not been examined (Zhang, Ho, \& Kennedy, 2014). The Transition Readiness Assessment Questionnaire (TRAQ) was identified as the best transition readiness assessment tool available, with good psychometric properties and a Likert-type rating scale for items rather than dichotomous "yes" or "no" responses (Sawicki et al., 2011; Zhang et al., 2014). T1DM patients, among others with pediatric health conditions, were included in two studies evaluating the TRAQ's psychometric properties (Sawicki et al., 2011; Wood et al., 2014). Results in both indicated that transition readiness is related to age, such that the older patients are, the readier they are to transition. It is important to note, however, that this measure asks for self- 
reported estimates of abilities, rather than measure objectively actual skill levels; consequently, it can be prone to bias in reporting.

Other factors have been identified as relevant to transition readiness. In one review, Monaghan and colleagues (2013) drew upon research in other pediatric populations to identify patient-provider communication as a potential variable of interest and point of intervention to improve transition readiness and health outcomes in T1DM. They also noted the relevance of systemic health care changes, such as legislation affecting insurance status, when considering transition readiness. In an effort to facilitate successful transition from pediatric to adult care for adolescents and young adults with T1DM, it is necessary to build upon this rather sparse literature base and investigate other factors that may be related to transition readiness.

\section{Health Literacy}

One fundamental variable that may be related to transition readiness in youth with T1DM is patient and parent health literacy. Health literacy is defined as "the degree to which individuals have the capacity to obtain, process, and understand basic health information and services needed to make appropriate health decisions" (U.S. Department of Health and Human Services, 2010). Notably, it is a construct that is distinct from basic literacy. Health literacy is context and content specific, meaning that those who have higher levels of general literacy may not be able to accurately employ their capabilities in unfamiliar contexts (e.g., health knowledge, health care settings; Nutbeam, 2009).

It is estimated that over one-third of adolescents and young adults have low health literacy (Sanders, Federico, Klass, Abrams, \& Dreyer, 2009). According to Manganello (2008), many systems impact health literacy, and health literacy impacts outcomes. The components of this model of health literacy are functional (i.e., ability to read and write in a health context), 
interactive (i.e., the combination of functional health literacy and social skills to communicate about health information effectively), critical (i.e., the ability to evaluate the quality of health information), and media (i.e., comprehension and evaluation of messages from the media) literacy. These forms of literacy may be studied in combination or alone (Manganello, 2008). Individual traits (e.g., demographic variables, media use) and family and peer factors affect health literacy. Similarly, health literacy and family and peer factors impact affect health outcomes (e.g., health behavior; Manganello, 2008). Consequently, according to this framework, health literacy interventions may result in a change in health outcomes, particularly in adolescents as they move toward independence in their disease management.

One-fourth of young adolescents in a school setting reported that it was hard to understand most of what they heard about health (Brown, Teufel, \& Birch, 2007). Those who reported that health information was difficult to understand were less likely to have interest in health information or follow what they were taught about making healthful decisions (Brown et al., 2007). School was cited as the key source of information about their health, although adolescents reported that they would go to parents, a health care professional, their school, and the Internet, respectively, if they had an important question about their health. Older children reported the Internet as a main source of health information; however, they believed that television and friends were the main sources of incorrect information, not the Internet (Brown et al., 2007). Qualitative data also suggest it is a challenge for adolescent students to access health information on the Internet due to health literacy difficulties (e.g., spelling search terms, describing symptoms) and determining the trustworthiness of websites (Gray, Klein, Noyce, Sesselberg, \& Cantrill, 2005). In a different sample of adolescents, recognizing a credible source of health information (MedlinePlus) was related to greater health literacy (Ghaddar, Valerio, 
Garcia, \& Hansen, 2012). These results imply that health literacy is important to obtaining and understanding reliable sources of health information; therefore, it is imperative to promote health literacy in youth in general, but particularly in those youths challenged with a chronic health condition.

Broadly, child and parent health literacy also are associated with health outcomes in child health settings (DeWalt \& Hink, 2009). For instance, parents with lower health literacy had less knowledge about weight-based dosing for liquid medication (Yin, Dreyer, Foltin, van Schaick, \& Mendelsohn, 2008). More research is needed, however, to determine the mechanism of these health literacy associations. Indeed, when health literacy is researched in pediatric populations, researchers most commonly measure solely parent health literacy levels. For example, in a large sample representative of parents in the United States, almost $30 \%$ had basic or below-basic health literacy (Yin et al., 2009). Almost half were unable to complete at least one of two medication-related tasks correctly, and those at below-basic health literacy levels were over three times more likely to have difficulty understanding over-the-counter medication labels (Yin et al., 2009). Parental health literacy also accounted for effects of some demographic variable-related disparities (e.g., education level, race/ethnicity, income; Yin et al., 2009). Thus, it is crucial to address low health literacy, targeting parent or provider behavior, to facilitate better parental decision-making capacity.

In addition to general populations, parental health literacy in T1DM has also been evaluated. In caregivers of children with T1DM, low parental health literacy has been related to suboptimal child health outcomes. For instance, caregivers who were classified as having inadequate health literacy had children (average age of 12 years old) with significantly poorer glycemic control than those of parents with higher health literacy levels (Hassan \& Heptulla, 
2010). In a population of high-risk adolescents with poor glycemic control, all parents were classified as having adequate health literacy (Janisse, Naar-King, \& Ellis, 2010). This was higher than trends in national data, potentially due to the availability of multidisciplinary providers, such as psychologists and diabetes educators (Janisse et al., 2010). For youth in this sample, higher reading comprehension levels were associated with better diabetes management behaviors (e.g., blood glucose monitoring) for adolescents prescribed an intensive insulin regimen (Janisse et al., 2010). In another study, lower caregiver diabetes numeracy was associated with poorer glycemic control in children three to nine years old with T1DM (Pulgarón et al., 2014). Notably, there was no significant relation among parental health literacy related to reading skills and HbA1c levels in this study (Pulgarón et al., 2014). All in all, parental health literacy appears to be related to health behaviors and outcomes for children and adolescents with T1DM.

In contrast to parent health literacy, research on health literacy in T1DM patients is scant. In particular, there is a significant gap in the literature when evaluating youth health literacy in relation to T1DM outcomes. In a study of adults with T1DM and type 2 diabetes, lower diabetesrelated numeracy was correlated with poorer self-management behaviors (Cavanaugh et al., 2008). Moreover, when controlling for demographic (e.g., age, sex) and diabetes-related (e.g., type, time since diagnosis) variables, better diabetes-related numeracy predicted better glycemic control (Cavanaugh et al., 2008). In adolescents with T1DM, better numeracy was significantly associated with better adherence to prescribed insulin pump use and greater glycemic control (Mulvaney, Lilley, Cavanaugh, Pittel, \& Rothman, 2013). These findings, in combination, support the apparent importance of mathematical skills applied to diabetes care and its healthrelated calculations, which is required multiple times per day in T1DM management (e.g., counting carbohydrates and subsequently making insulin adjustments). Parental written health 
literacy skills may not be as relevant, although this has not been investigated across all youth ages, or at all in youth (e.g., Pulgarón et al., 2014). Considering the relation of adolescent and parental health literacy to numerous diabetes self-management behaviors and health outcomes also important to transition readiness, health literacy is a logical direction for future work about transition readiness in youth with T1DM.

\section{Responsibility}

Given relatively young age of T1DM diagnosis, family factors play a significant role in youth outcomes and likely are associated with transition readiness in adolescents. The Diabetes Family Responsibility Questionnaire (DFRQ) was created to examine how youth with T1DM share responsibility for disease management tasks with their mothers (Anderson, Auslander, Jung, Miller, \& Santiago, 1990). Quantitative and qualitative literature indicates that greater youth age is associated with greater youth responsibility for T1DM-related tasks, meaning that older children take more responsibility for managing their disease (Anderson et al., 1990; Ingerski, Anderson, Dolan, \& Hood, 2010; Schilling, Knafl, \& Grey, 2006). Furthermore, female youth aged 6 to 21 years old reported taking more responsibility than males (Anderson et al., 1990).

Due to the shared nature of diabetes management in youth, discrepancies in reported responsibility may be cause for concern. Gaps in reported responsibility have been found to be associated with glycemic control (HbA1c; Anderson et al., 1990). Specifically, when motherand child-report about responsibility for tasks are compared, they may each respond that responsibility for the task is allocated to the other person, indicating that there is a gap in responsibility. Greater reported mismatches in responsibility and lower mother-reported adherence significantly predicted lower HbA1c (Anderson et al., 1990). 
Contrary to disagreements in division of responsibility, parents and youth also report sharing responsibility for a number of T1DM management tasks. The importance of agreement was demonstrated in more recent research (Lancaster, Gadaire, Holman, \& LeBlanc, 2015). In a study of youth (ages 8-18 years) and their parents, greater agreement of responsibility allocation was a predictor of better glycemic control. Similarly, greater shared parent-child responsibility is associated with better HbA1c (Follansbee, 1989). Additionally, adolescents who report greater shared responsibility with their parents in tasks directly managing their T1DM tend to monitor their blood glucose more often (Vesco et al., 2010). These findings are consistent with those of another study demonstrating that excessive self-care by adolescents is related to poorer treatment adherence (Wysocki et al., 1996). Evaluating the agreement and gaps in responsibility for diabetes care tasks may provide greater insight into the T1DM management patterns of children and their caregivers.

Like trends found in samples of other pediatric chronic health conditions, parents tend to surrender control and expect greater responsibility for youth to self-manage their diabetes as they age into adolescence (Sawicki et al., 2011). Specifically, research shows that parents participate less in the self-management task of insulin adjustment as their adolescent grows older (Ingersoll, Orr, Herrold, \& Golden, 1986). In fact, most parents relinquish responsibility for insulin administration by the time their adolescents are 15 years old, expecting adolescents to be independent; yet, adolescents do not always assume complete responsibility by this time (Ingersoll et al., 1986). More cognitively mature youth are more likely to take responsibility for insulin adjustment and have greater glycemic control (Ingersoll et al., 1986). Therefore, it is important to consider adolescent preparedness before removing parental involvement in selfmanagement tasks. 
Sullivan-Bolyai and colleagues (2014) allowed adolescents with T1DM and their parents to share their perspectives about disease management via focus groups. Adolescents who experienced an early onset of symptoms reported that they did not know what it was like to live without T1DM, while others reported variations in eagerness to learn about T1DM. They also reported that peer support groups and attending a diabetes camp were not helpful, though they expressed interest in mentoring younger T1DM patients. The difficulty of transitioning responsibility to the adolescent was a major theme in the parent groups (Sullivan-Bolyai et al., 2014). Some especially reported that they felt provider pressure to take responsibility for care, but believed that their responsibility was to facilitate adolescent autonomy. In another qualitative study, parents reported that it was difficult to trust adolescents to manage their regimen (Ivey, Wright, \& Dashiff, 2009). Some reported feelings of helplessness and loss of control to an adolescent they viewed as unprepared to take full responsibility. Parents also reported that they believed their adolescents may be dishonest about self-management behaviors (Ivey et al., 2009). Other themes from this research included parental fear of adolescents mismanaging their disease, as well as adolescent and parental frustration toward one another related to T1DM management tasks (Ivey et al., 2009). This frustration and lack of proper communication, particularly around transfer of responsibility, may result in increased conflict between family members and the adolescent. As adolescents approach an age where transition to adult care is imminent, this transfer of responsibility and potential conflict is particularly salient.

\section{Family Conflict}

Family conflict for pediatric patients with T1DM often can center around disease management. Scores on the Diabetes Family Conflict Scale-Revised (DFCS-R), a scale of diabetes-specific family conflict, have been significantly correlated with HbA1c levels, 
indicating that greater conflict is associated with poorer glycemic control (e.g., Hood, Butler, Anderson, \& Laffel, 2007). Furthermore, conflict related to direct management tasks (e.g., remembering to check blood sugars) was a better predictor of glycemic control than indirect management tasks (e.g., telling teachers about diabetes), suggesting that conflict about direct management tasks is more important to consider as youth become more autonomous and begin the transition process. Qualitatively, adolescent and parent dyads reported most frequent conflict around food choices and blood glucose testing (Schilling et al., 2006). Adolescents with poorer glycemic control reported more annoyance and conflict with their parents about treatment management, as well (Leonard, Garwick, \& Adwan, 2005). Similarly, in adolescents with T1DM, better reported conflict resolution skills and communication within families were predictive of better glycemic control and greater adherence to multiple tasks (e.g., injections, blood glucose monitoring; Miller-Johnson et al., 1994; Wysocki, 1993). Greater diabetes-specific family conflict also predicted later increases in HbA1c levels, meaning poorer blood glucose control (Ingerski et al., 2010). Notably, in the same study, youth reported greater levels of conflict than their parents did, indicating that adolescents and their parents may perceive communication about T1DM management tasks differently (Ingerski et al., 2010). Furthermore, when included with parent-child agreement of responsibility for T1DM management tasks, conflict related to diabetes significantly predicted HbA1c levels (Lancaster et al., 2015).

Anderson and colleagues (2002) proposed some hypotheses for the link between conflict and glycemic control. Increased conflict may result in higher glucose levels as a result of increased stress hormones. Alternatively, parent-child conflict may increase in reaction to poor glycemic control. Overall, these findings demonstrate that family conflict about diabetes 
management tasks is highly relevant to glycemic control and health outcomes in children and adolescents.

Given the apparent concerns of diabetes-specific family conflict (e.g., Hood et al., 2007), Behavioral Family Systems Therapy (BFST) was developed and subsequently tested in families of adolescents with T1DM (Wysocki et al., 2000). In BFST, conflict results from the adolescent's desire for autonomy in contrast to the parent's desire to control and remain involved. When compared to an education and support group and treatment as usual, participants receiving BFST experienced decreases in diabetes-specific conflict, though there were no differences in health outcomes (Wysocki et al., 2000). This improvement in conflict was maintained long-term up to one year, and delayed positive effects on treatment adherence were also observed at 6- and 12-months post-treatment (Wysocki, Greco, Harris, Bubb, \& White, 2001). To target T1DM health outcomes, BFST for Diabetes (BFST-D) was created (Wysocki et al., 2007). Unlike the original BFST, the BFST-D group had a significantly better impact on glycemic control, mediated by better adherence to treatment (Wysocki et al., 2007). There was no change in parent-child conflict, though. Despite the lack of change in diabetes-specific family conflict in this program, the research on BFST revealed that conflict could indeed be targeted through intervention.

Along these same lines, Laffel and colleagues (2003) conducted a study comparing the effects of a family-focused teamwork intervention to standard care in youth with T1DM. The intervention included modules on communication skills related to diabetes and working together to share the burden of T1DM management. Unlike for youth in the control group, HbA1c levels did not deteriorate for the intervention group (Laffel et al., 2003). Additionally, the intervention group had a significantly greater number of families being involved at levels similar to or greater 
than baseline. Even with greater parental involvement, families in the intervention group did not report an increase in conflict (Laffel et al., 2003). This demonstrated a successful intervention targeting parent-child cooperation related to T1DM management during a time period when behavioral and health outcomes decline. Increased parental involvement with age is not ideal, however, as autonomously managing T1DM is the goal for preparing to transition. No longitudinal research has been conducted on the process of fading parental involvement to promote autonomy. More research is necessary to determine which parent behaviors facilitate effective, autonomous self-management and as a result, transition readiness.

\section{Supportive Parental Behaviors}

Along with characterizing parent-child interactions such as diabetes-related conflict, specific parental behaviors may facilitate or hinder transfer of responsibility, transition of health care, and health outcomes in adolescents with T1DM. For instance, consider supportive (e.g., parental praise for child adherence) and non-supportive (e.g., nagging/criticizing about selfmanagement tasks) behaviors, as measured by the Diabetes Family Behavior Checklist (DFBC; Lewin et al., 2005). Child and parent report of non-supportive behaviors have been correlated with lower glycemic control and adherence (Lewin et al., 2005). Furthermore, parent report of supportive behaviors has been associated with better HbA1c levels (Lewin et al., 2005). Similarly, child reported supportive behaviors were correlated with greater adherence (Lewin et al., 2005). These supportive behaviors combined with other family factors (e.g., responsibility) and adherence also significantly predicted glycemic control (Lewin et al., 2006). Additionally, more parental guidance and control behaviors, such as providing reminders for and observing blood glucose monitoring, were associated with better glycemic control in youth (McKelvey et al., 1993). Overall, these results have implications for the importance of the family context of 
disease management in T1DM. Given the relation that parental support behaviors have shown to T1DM management in youth, it seems logical to assume that parental support might play a significant role in promoting transition readiness. However, this area of research has not yet been explored. Indeed, many of these relevant parental and family constructs (e.g., family conflict, parental support) have not been evaluated in relation to transition programs or readiness; thus, more research is necessary to reveal the role of family factors in transition.

\section{Summary and Rationale for Current Study}

In summary, T1DM is a complicated health condition that can be burdensome to manage. Daily self-management tasks include checking blood glucose levels, calculating and administering the correct doses of insulin, eating the appropriate amount of carbohydrates, and engaging in physical activity (ADA, 2015a; ADA, 2015b; JDRF, 2017). Maintaining consistently healthy blood glucose levels is a primary goal to prevent potentially life-threatening complications (JDRF, 2017; The Diabetes Control and Complications Trial Research Group, 1993). This goal is assessed long-term via glycated hemoglobin values (HbA1c; Jeffcoate, 2004) during clinic appointments.

Considering the complexities of T1DM treatment, adolescence through young adulthood is an important period of the life span to consider. This is the time when patients begin to take on more autonomy in their care as well as the active process of transitioning from pediatric to adult care providers and settings. In addition to identity exploration and change during emerging adulthood, glycemic control becomes worse during this developmental phase due, in part, to behavioral factors such as suboptimal insulin treatment adherence and non-attendance of clinic appointments (Arnett, 2000; Jacobson et al., 1997; Morris et al., 1997). 
There are multiple factors that theoretically influence transition readiness and health outcomes, including patient skills and family system variables (Pierce \& Wysocki, 2015). Despite the complicated nature of transition readiness in T1DM, there is a dearth of research on the topic. In adolescents with T1DM, when included in samples of youth with other chronic conditions, a positive association with age and preparedness for transition has been observed (Sawicki et al., 2011; Wood et al., 2014). Patient-provider communication has been identified as a potential influence on transition readiness, as well (Monaghan et al., 2013). Considering the expanded SMART framework, there can be other variables that are related to transition readiness; yet, more research is needed to identify these constructs (Pierce \& Wysocki, 2015). Health literacy appears to be a skill that is pertinent to transition readiness and health outcomes in adolescents with T1DM. Health literacy may be particularly relevant to these outcomes considering the complexity of T1DM management, for which the ability to understand and apply written and complicated mathematical calculations is essential. According to Manganello's (2008) theoretical model, functional health literacy and family factors influence outcomes. In samples of caregivers of youth with T1DM, greater caregiver health literacy was associated with superior health outcomes (e.g., Janisse et al., 2010; Pulgarón et al., 2014). A main limitation of the research on the association between health literacy, as well as behavior and health outcomes, is that most all studies focus on parental health literacy. In contrast, however, one study examining adolescent health literacy and T1DM outcomes, found that better math-related health literacy was associated with superior self-management behavior and glycemic control (Mulvaney et al., 2013). This leaves numerous gaps in the research on both written and mathematical aspects of adolescent health literacy, as these are important skills that may predict transition readiness and health outcomes. 
Family factors may also be relevant to transition readiness due to their clear role in other outcomes in youth with T1DM. Specifically, the allocation of responsibility for T1DM tasks differs based on age, with older adolescents reporting that they take more responsibility for disease management than younger adolescents (e.g., Anderson et al., 1990; Sawicki et al., 2011; Schilling et al., 2006). Although autonomous self-management is necessary for successful transition to adolescent care, no literature exists about the division of disease management tasks and transition readiness, indicating the need for future investigation. Furthermore, due to the significant effort required to manage T1DM, families may experience diabetes-specific conflict, particularly in adolescence. Indeed, conflict related to diabetes has implications for glycemic control, specifically that lower conflict predicts better HbA1c levels (e.g., Hood et al., 2007; Ingerski et al., 2010). Good glycemic control is also related to behaviors such as adherence to disease management tasks (e.g., Miller-Johnson et al., 1994). Again, although diabetes-specific family conflict appears to be relevant to behavior and health outcomes, it is unknown how it may relate to adolescent transition readiness. Similarly, certain supportive and non-supportive parental behaviors are associated with vital behaviors for proper T1DM management in addition to glycemic control (e.g., Lewin et al., 2005). In accordance with these findings, it is logical that specific parental support behaviors may promote transition readiness skill attainment, but this research has not been conducted yet in T1DM. Therefore, the overall objective of this project was to examine family factors as they interact with health literacy and subsequently relate to transition readiness and long-term glycemic control. As a result, the aims of the current project were:

Aim 1. The first aim of the study was to examine the association of adolescent health literacy with transition readiness and with health outcome (i.e., glycemic control) in adolescents 
with T1DM. Based on research in adolescents and caregivers of youth with T1DM, it was hypothesized that adolescent health literacy would be significantly and positively correlated with glycemic control (e.g., Mulvaney et al., 2013; Pulgarón et al., 2014). Because the relation between adolescent health literacy and transition readiness had not yet been investigated, the hypothesis that they would be significantly and positively associated was based on clinical judgment.

Aim 2. The second aim of the project was to explore the extent to which family factors (i.e., diabetes management responsibility, family conflict, parental supportive behavior) served as moderators in adolescent health literacy predicting transition readiness and health outcome in T1DM (i.e., glycemic control; see Figure 2). Because there was no literature examining the relation amongst adolescent health literacy, these particular family factors, and transition readiness or glycemic control, no hypothesis was created.

\section{Method}

\section{Participants}

Seventy patients with T1DM and their primary caregivers were enrolled. Patients were recruited from the Pediatric Endocrinology clinics at the West Virginia University Physicians' Office Center during an appointment for a routine follow-up or for a presenting complaint. Included in the sample were patients who: a) were 13 to 17 years old; b) had been diagnosed with T1DM for at least six months; and c) had a primary caregiver who agreed to participate. Patients who could not complete the study measures due to language barriers or cognitive deficits, as judged by a member of the healthcare or research team, were excluded from recruitment. Five dyads were excluded from final data analyses: one participant departed from the visit early due to medical complications, and four participants had greater than $10 \%$ missing 
data on a measure. Characteristics of the study sample $(N=65)$ appear in Table 1 . The mean patient age was 15.03 years $(S D=1.49$, Range $=13-17)$, and $36(55 \%)$ were male. The mean duration of diagnosis was 6.15 years $(S D=3.97$, Range $=1-16)$. Most patients identified as Caucasian, Non-Hispanic (95\%), and most caregivers participating in the study were biological mothers $(80 \%)$.

\section{Procedure}

Eligible patients were identified by the healthcare team and approached during their appointments to determine if they were interested in learning more about the study. Once families agreed, undergraduate and/or graduate-level members of the research team explained the study purpose, procedure, benefits, risks, confidentiality, and HIPPA policies. Assent was obtained from all patients, and consent was obtained from all primary caregivers. After assent and consent were obtained, participants completed questionnaires on tablets using Research Electronic Data Capture (REDCap). Patients completed two health literacy measures (TOFHLA$R, D N T-14)$, a transition readiness survey $(T R A Q)$, three diabetes-family measures $(D F R Q$, $D F C S-R$, and $D F B C-C$ ), and a diabetes knowledge measure (DKT2). Caregivers completed the Family Information Form, and the patient's most recent HbAlc value was recorded from medical records. Each family received a \$20 gift card upon completion.

\section{Measures}

Family Information Form. The Family Information Form is a questionnaire developed for this study to obtain relevant demographic and medical information for the patient and family (e.g., age, race, education level, and medical history). Caregivers completed this form.

Test of Functional Health Literacy in Adults (TOFHLA; Parker, Baker, Williams, Nurss, 1995). The TOFHLA is a test of functional health literacy that measures reading 
comprehension (TOFHLA-R) and numeracy $(T O F H L A-N)$. The reading comprehension component has three health-related passages with 50 total words omitted (e.g., "After , you must not or drink"). For each blank, participants select one response from a choice of four to correctly complete the sentence. A total score is obtained by calculating the percentage of correct responses. Higher scores indicate greater functional health literacy. In a sample of adults, the TOFHLA scores demonstrated excellent internal consistency (Cronbach's $\alpha=.98$; Chisolm \& Buchanan, 2007). TOFHLA-R scores have been validated in an adolescent population with significant correlations to other assessments of literacy (Chisolm \& Buchanan, 2007). Nonetheless, TOFHLA-N scores were not determined to be valid in the same adolescent population (Chisolm \& Buchanan, 2007); therefore, this measure was not used to assess functional numeracy skills in this project. The TOFHLA-R was completed by adolescents to assess written health literacy. Although lower than a sample of adults for the overall measure, internal consistency for the current adolescent sample was good (Cronbach's $\alpha=.84$ ).

\section{Diabetes Numeracy Test-14 (DNT-14; Mulvaney, Lilley, Cavanaugh, Pittel, \&}

Rothman, 2013). The DNT-14 is a measure of diabetes-specific numeracy in adolescents. It was developed from the Diabetes Numeracy Test (DNT; Huizinga et al., 2008). The DNT-14 consists of 14 free response items assessing math skills (e.g., addition, division, multi-step mathematics, time) across content areas including nutrition and blood glucose monitoring (e.g., "You test your blood sugar 4 times a day. How many strips do you need to take with you on a 2-week vacation?"). Participants may use extra paper and a calculator if desired. A total score is obtained by calculating the percentage of correct responses. Higher scores indicate greater numeracy skills. In a population of adolescents with T1DM, scores had good internal reliability $(\mathrm{KR} 20=$ .83; Mulvaney et al., 2013). It also demonstrated good predictive validity, as greater diabetes 
numeracy was associated with better glycemic control (Mulvaney et al., 2013). Youth completed this measure to assess numeric health literacy. Internal reliability for this sample was good $(\mathrm{KR} 20=.80)$.

Transition Readiness Assessment Questionnaire (TRAQ; Wood et al., 2014). The $T R A Q$ is a measure of self-management skills used to prepare for transition from pediatric to adult care; it is generic, rather than diabetes-specific. The initial measure included 29 items, although recent item reduction has demonstrated support for a 20-item scale (Sawicki et al., 2011; Wood et al., 2014). The 20-item version consists of five subscales: Appointment Keeping (e.g., "Do you call the doctor's office to make an appointment?"), Managing Medications (e.g., “Do you fill a prescription if you need to?”), Talking with Providers (e.g., "Do you answer questions that are asked by the doctor, nurse, or clinic staff?”), Tracking Health Issues (e.g., "Do you make a list of questions before the doctor's visit?’), and Managing Daily Activities (e.g., “Do you help plan or prepare meals/food?"). Adolescents rate each item on a scale of 1 (“I do not need to do this") to 5 ("I always do this when I need to") corresponding to the Stages of Change Model (Prochaska \& DiClemente, 1986). Total and subscale scores result from averaging item scores within the subscales. Higher scores indicate greater transition readiness. In a population of adolescents with special health care needs, internal consistency for the total score was excellent (Cronbach's $\alpha=.94$ ), and subscale reliability (Cronbach's alpha) ranged from .67 to .90 (Wood et al., 2014). Scores have demonstrated criterion-related validity with respect to age, meaning greater transition readiness is associated with higher age (Wood et al., 2014). Youth completed this measure of transition readiness, and the total score was used in analyses. Internal consistency for this sample was excellent (Cronbach's $\alpha=.92$ ). 


\section{Diabetes Family Responsibility Questionnaire (DFRQ; Anderson et al., 1990; Vesco et}

al., 2010). The $D F R Q$ is a youth- and parent-report assessment of treatment management responsibility perceptions. The version validated in adolescents by Vesco and colleagues (2010) was utilized. It consists of 17 items across two domains: Direct (e.g., "Deciding what to eat at meals or snacks") and Indirect (e.g., "Telling relatives about diabetes”) Management. Respondents rate each item on a scale of 1 ("adolescent takes or initiates responsibility for this almost all of the time") to 3 ("parent[s] take or initiate responsibility for this almost all of the time”). Scores are summed and range from 17 (adolescent has complete responsibility) to 51 (parent has complete responsibility), with a score of 34 meaning that the adolescent and parent

share responsibility equally. In a population of adolescents with T1DM, internal consistency was acceptable (Cronbach's $\alpha=.74$; Vesco et al., 2010). To assess construct validity, a confirmatory factor analysis produced a two-factor solution consistent with direct and indirect management tasks (Vesco et al., 2010). The direct management factor score had acceptable internal consistency (Cronbach's $\alpha=.73$ ), while the indirect management factor score had lower internal consistency (Cronbach's $\alpha=.57$; Vesco et al., 2010). For this study, however, the adolescentreport total score was used in analyses. Internal consistency for the total score with this sample was good (Cronbach's $\alpha=.89$ ).

Diabetes Family Conflict Scale-Revised (DFCS-R; Hood et al., 2007). The DFCS-R is a youth- and parent-report measure of conflict frequency related to diabetes management tasks. It is a 19-item revision of the DFCS (Rubin, Young-Hyman, \& Peyrot, 1989). The DFCS-R instructs respondents to rate how frequently they have argued with their parent(s) about various diabetes management tasks over the past month on a scale of 1 ("almost never") to 3 ("almost always"). There are two subscales: Direct (e.g., "logging blood sugar results") and Indirect (e.g., 
"what to eat when away from home"). Total and subscale scores are calculated by summing item responses, with higher scores indicating greater family conflict with respect to diabetes management. In a population of youth with T1DM, internal consistency was good (Cronbach's $\alpha$ $=.85$ ), and subscale reliability (Cronbach's alpha) was .69 and .75 (Hood et al., 2007). Scores also demonstrate concurrent validity with health-related quality of life and negative affect related to blood glucose monitoring (Hood et al., 2007). Furthermore, greater diabetes-related family conflict is significantly predictive of poorer glycemic control. Youth completed this questionnaire as a measure of diabetes-related family conflict. The total score was used in analyses. Cronbach's alpha (.96) was excellent for this score in the current sample.

Diabetes Family Behavior Checklist (DFBC; Schafer et al., 1986). The DFBC is a youth- $(D F B C-C)$ and parent- $(D F B C-P)$ report measure of supportive and non-supportive family interaction frequency. It instructs respondents to rate how often family members engage in behaviors related to diabetes care across two subscales: Supportive (e.g., "plan family activities so that they will fit in with his/her diabetes self-care schedule") and Non-Supportive (e.g., "eat foods that are not part of the patient's diabetic diet;" Lewin et al., 2005). Response options range from 1 ("never") to 5 (“at least once a day"). Subscale scores are calculated by summing item responses, with higher scores indicating greater behavior frequency. In a study of youth with T1DM (Lewin et al., 2005), internal consistency was acceptable for the Supportive (Cronbach's $\alpha=.74$ ) and Non-Supportive (Cronbach's $\alpha=.79$ ) subscales. In the current study, adolescents completed this measure to assess supportive and non-supportive caregiver behaviors. The internal consistency for this sample was similarly acceptable for the Supportive (Cronbach's $\alpha=.78$ ) and Non-Supportive (Cronbach's $\alpha=.72$ ) subscales. 
Revised Brief Diabetes Knowledge Test 2 (DKT2; Fitzgerald et al., 2016). The DKT2 is a 23-item assessment of knowledge about diabetes and insulin use. It has a multiple-choice format with respondents selecting one answer for each question. The DKT2 has a fourth-grade reading level. In a study of adults with types 1 and 2 diabetes, its scores demonstrated acceptable reliability (Cronbach's $\alpha=.77$ ) for the general knowledge subscale and good reliability (Cronbach's $\alpha=.84$ ) for the insulin use subscale (Fitzgerald et al., 2016). However, results using this measure have not yet been published in an adolescent sample. A total score (percentage of answers answered correctly) was used in analyses. Adolescents completed this measure to assess general diabetes knowledge. Cronbach's alpha for the current sample was .66, which is acceptable, yet lower than previously reported samples.

HbA1c. HbA1c values measure blood glucose levels over approximately the past two to three months (Jeffcoate, 2004). Values were obtained from routine blood tests completed at clinic appointments; the most recent value was extracted from the patient's medical record. Current guidelines recommend that youth with T1DM have HbA1c less than 7.5\% (ADA, 2017), with higher values indicating poorer glycemic control.

\section{Results}

Statistical analyses were conducted using Statistical Package for the Social Sciences version 25 (SPSS 25) and PROCESS macro.

\section{Power Analysis}

An a priori power analysis was conducted to guide plans for recruitment. The sample size was calculated using $\mathrm{G}^{*}$ Power based on the aim with the largest number of predictors (Aim 2). To detect a medium effect size with alpha of .05 and $80 \%$ power, a sample of 77 dyads was required. To detect a large effect size with alpha of .05 and $80 \%$ power, a sample of 36 dyads is 
required. Consequently, this study was powered to detect medium to large effect sizes by recruiting 70 dyads.

\section{Preliminary Analyses}

Missing data were identified and determined to be missing completely at random utilizing Little's MCAR test. Five participants had greater than $10 \%$ of items missing responses on at least one measure. All five participants' missing data included independent and/or outcome variables; therefore, these participants were deleted listwise from analyses. For participants with less than $10 \%$ missing data $(n=65)$, multiple imputation was used to impute missing data values. Outliers labeled as extreme values in SPSS were identified. To address these outliers, the following variable scores were winsorized (substituted with the next highest non-outlier score): DKT2, TOFHLA-R, DNT-14, and DFRQ. All other variable distributions were normal after winsorizing. Data did not violate the assumption of multicollinearity, as the variance inflation factors (VIF) ranged from 1.14 to 1.60 .

Descriptive statistics for raw primary variables are displayed in Table 2. Pearson correlation coefficients for adjusted variables are presented in Table 3. Diabetes knowledge was significantly correlated with both measures of health literacy, such that greater diabetes knowledge was associated with greater numeric $[r(65)=0.45, p<.01]$ and written $[r(65)=0.44$, $p<.01]$ health literacy. DKT2 scores were included as a covariate for analyses reported in the Appendix. Numeric and written health literacy also were significant positively correlated, $r(65)=$ $0.43, p<.01$. Higher written health literacy was significantly associated with higher parental responsibility $[r(65)=0.31, p=.02]$ and lower family conflict $[r(65)=-0.27, p=.01]$. Higher parental responsibility was also significantly correlated with fewer parental supportive behaviors $[r(65)=-0.30, p=.02]$ and greater transition readiness $[r(65)=0.31, p=.03]$. Greater parental 
supportive diabetes-related behaviors were significantly associated with more frequent parental non-supportive behaviors $[r(65)=0.33, p=.01]$. Correlation coefficients (i.e., Pearson or Spearman) relating demographic variables to predictor and outcome variables are presented in Table 4. Greater age was significantly correlated with greater reported parental responsibility $[r(65)=0.31, p=0.01]$ and greater transition readiness $[r(65)=0.42, p<0.01]$. Higher caregiver education was significantly associated with greater diabetes numeracy in youth $[\rho(65)=0.25, p=$ 0.04]. Greater family income was significantly correlated with lower blood glucose $[\rho(65)=-$ $0.30, p=0.01]$. Furthermore, private insurance was associated with fewer non-supportive family behaviors $[\rho(65)=-0.34, p=0.01]$ and lower blood glucose $[\rho(65)=-0.37, p<0.01]$.
$\operatorname{Aim} 1$
The first aim was to examine the association of adolescent health literacy with transition readiness and health outcomes. Pearson correlation coefficients were calculated between health literacy (individual TOFHLA-R and DNT-14) and transition readiness (TRAQ) scores, as well as glycemic control index (HbA1c). A significant relation was not found among written health literacy and transition readiness, $r(65)=-0.10, p=.50$, nor written health literacy and glycemic control, $r(65)=-0.05, p=.43$. There was no significant relation among numeric health literacy and transition readiness, $r(65)=0.10, p=.43$, nor was there a significant relation among numeric health literacy and glycemic control, $r(65)=-0.22, p=.08$.

\section{$\operatorname{Aim} 2$}

The second aim was to explore the extent to which family factors (i.e., diabetes management responsibility, family conflict, parental supportive behavior) served as moderators in health literacy predicting transition readiness and health outcome in T1DM. To evaluate this aim, each family factor $(D F R Q, D F C S-R$, and $D F B C$ - $C$ supportive behaviors, and $D F B C$ - $C$ non- 
supportive behaviors) was tested as a potential moderator for individual health literacy measures (TOFHLA-R and DNT-14) predicting (a) transition readiness (TRAQ), and (b) glycemic control (HbA1c) in separate models. Parallel multiple linear regression models were run, one each for the separate measures of health literacy as the independent variable; both models included individual family factors as the moderator and the health literacy $\mathrm{X}$ family factor interaction.

Written health literacy predicting transition readiness. With written health literacy predicting transition readiness, the overall model with diabetes management responsibility was significant, $F(3,61)=3.84, p=.01, R^{2}=.16($ see Table 5$)$. Written health literacy was not a significant predictor of transition readiness, $b=-4.22, p=.18,95 \%$ CI [-10.46, 2.03], nor was responsibility a significant predictor of transition readiness, $b=-2.81, p=.46,95 \% \mathrm{CI}[-10.31$, 4.68]. There was no significant moderation, as the written health literacy $\mathrm{X}$ responsibility interaction did not significantly predict transition readiness, $b=0.09, p=.30,95 \%$ CI $[-0.08$, $0.26]$.

The overall moderation model with family conflict as a proposed moderator of written health literacy and transition readiness was not significant, $F(3,61)=1.40, p=.25, R^{2}=.06$ (see Table 5). Written health literacy did not significantly predict transition readiness, $b=2.19, p=$ $.21,95 \%$ CI $[-1.23,5.62]$. Family conflict did not significantly predict transition readiness, $b=$ $3.44, p=.11,95 \%$ CI $[-0.76,7.65]$. The written health literacy $\mathrm{X}$ family conflict interaction term was not a significant predictor of transition readiness, $b=-0.08, p=.09,95 \%$ CI [-0.18, 0.01], resulting in no significant moderation.

The model with parental supportive behavior moderating written health literacy and transition readiness was not significant, $F(3,61)=0.46, p=.71, R^{2}=.02$ (see Table 5). Neither written health literacy, $b=0.36, p=.86,95 \%$ CI $[-3.62,4.34]$ nor supportive behavior, $b=1.43$, 
$p=.64,95 \%$ CI $[-4.33,7.02]$ significantly predicted transition readiness. Written health literacy

$\mathrm{X}$ supportive behavior did not significantly predict transition readiness, $b=-0.03, p=.69,95 \%$

CI [-0.16, 0.11]; thus, moderation was not present.

The overall model for parental non-supportive behaviors moderating the relation of written health literacy and transition readiness was not significant, $F(3,61)=1.81, p=.16, R^{2}=$ .08 (see Table 5). Written health literacy did not significantly predict transition readiness, $b=$ $2.91, p=.12,95 \%$ CI $[-0.75,6.56]$. Non-supportive family behavior did not significantly predict transition readiness, $b=7.95, p=.05,95 \%$ CI $[-0.10,16.00]$. The written health literacy X nonsupportive behavior moderation term was not significant, $b=-0.18, p=.06,95 \%$ CI [-0.36, 0.01], indicating moderation was not present.

Written health literacy predicting glycemic control. The overall moderation model with responsibility as a proposed moderator of written health literacy and glycemic control was not significant, $F(3,61)=0.21, p=.89, R^{2}=.01$ (see Table 6$)$. Neither written health literacy, $b=$ $-0.09, p=.81,95 \%$ CI [-0.85, 0.67], nor responsibility, $b=-0.04, p=.94,95 \%$ CI [-0.95, 0.88], significantly predicted glycemic control. The written health literacy X responsibility interaction term was not a significant moderator, $b=0.00, p=.89,95 \%$ CI $[-0.02,0.02]$.

The model with family conflict moderating written health literacy and glycemic control was not significant, $F(3,61)=0.65, p=.58, R^{2}=.03$ (see Table 6$)$. Written health literacy did not significantly predict glycemic control, $b=-0.26, p=.19,95 \%$ CI [-0.65, 0.13]. Family conflict did not significantly predict glycemic control, $b=-0.30, p=.21,95 \%$ CI $[-0.78,0.17]$. Moderation was not present, as the written health literacy $\mathrm{X}$ family conflict term was not significant, $b=0.01, p=.20,95 \%$ CI $[0.00,0.02]$. 
The overall moderation model with parental supportive behavior as a proposed moderator of written health literacy and glycemic control was not significant, $F(3,61)=0.88, p=.45, R^{2}=$ .04 (see Table 6). Written health literacy predicting glycemic control was not significant, $b=$ $0.08, p=.73,95 \%$ CI $[-0.37,0.52]$. Supportive behavior was not a significant predictor of glycemic control, $b=0.11, p=.72,95 \% \mathrm{CI}[-0.52,0.74]$. The written health literacy X parental supportive behavior interaction was not significant, $b=0.00, p=.62,95 \%$ CI [-0.02, 0.01$]$; thus, moderation was not present.

With glycemic control as the outcome, the overall model with parental non-supportive behavior as moderator was not significant, $F(3,61)=0.77, p=.51, R^{2}=.04$ (see Table 6$)$. Neither written health literacy, $b=0.01, p=.95,95 \%$ CI [-0.41, 0.43], nor parental nonsupportive behavior, $b=0.12, p=.80,95 \%$ CI $[-0.81,1.04]$, significantly predicted glycemic control. There was no significant moderation, as the written health literacy X non-supportive behavior interaction was not a significant predictor of glycemic control, $b=0.00, p=.88,95 \%$ CI $[-0.02,0.02]$.

Numeric health literacy predicting transition readiness. The overall model with family responsibility moderating the relation between numeric health literacy and transition readiness was significant, $F(3,61)=3.15, p=.03, R^{2}=.13$ (see Table 7). However, the individual predictors in this model were not significant. Numeric health literacy did not significantly predict transition readiness, $b=-5.78, p=.15,95 \%$ CI [-13.78, 2.23]. Responsibility did not significantly predict transition readiness, $b=-0.86, p=.47,95 \%$ CI [$3.22,1.51]$. The numeric health literacy $\mathrm{X}$ responsibility moderation term was not significant, $b$ $=0.16, p=.13,95 \% \mathrm{CI}[-0.05,0.37]$. 
The model with family conflict moderating numeric health literacy and transition readiness was not significant, $F(3,61)=0.52, p=.67, R^{2}=.02$ (see Table 7). Neither numeric health literacy, $b=2.37, p=.28,95 \%$ CI $[-1.96,6.70]$, nor family conflict, $b=0.53, p=.44$, $95 \%$ CI $[-0.82,1.87]$, were significant predictors of transition readiness. Moderation was not present, as the numeric health literacy $\mathrm{X}$ family conflict interaction term did not significantly predict transition readiness, $b=-0.06, p=.36,95 \%$ CI $[-0.18,0.07]$.

The overall model with parental supportive behavior as a proposed moderator of the relation between numeric health literacy and transition readiness was not significant, $F(3,61)=$ $0.60, p=.61, R^{2}=.03$ (see Table 7). Numeric health literacy did not significantly predict transition readiness, $b=-1.49, p=.61,95 \%$ CI [-7.28, 4.29]. Supportive behavior did not significantly predict transition readiness, $b=-0.51, p=.62,95 \% \mathrm{CI}[-2.55,1.53]$. The numeric

health literacy X supportive behavior interaction did not significantly predict transition readiness, $b=0.07, p=.47,95 \%$ CI $[-0.12,0.25]$; therefore, moderation was not present.

With transition readiness as the outcome, the overall moderation model with parental non-supportive behavior moderating numeric health literacy was not significant, $F(3,61)=0.93$, $p=.43, R^{2}=.04$ (see Table 7). Numeric health literacy did not significantly predict transition readiness, $b=2.45, p=.23,95 \%$ CI $[-1.55,6.45]$, nor did non-supportive behavior significantly predict transition readiness, $b=1.40, p=.20,95 \% \mathrm{CI}[-0.75,3.55]$. The numeric health literacy $\mathrm{X}$ parental non-supportive behavior interaction did not significantly moderate the relation between numeric health literacy and transition readiness, $b=-0.10, p=.30,95 \%$ CI $[-0.30$, 0.09].

Numeric health literacy predicting glycemic control. The model with family responsibility moderating the relation between numeric health literacy and glycemic control was 
not significant, $F(3,61)=1.35, p=.27, R^{2}=.06$ (see Table 8$)$. Neither numeric health literacy, $b$ $=0.03, p=.94,95 \%$ CI [-0.90, 0.97], nor responsibility, $b=0.09, p=.53,95 \%$ CI [-0.19, 0.36], significantly predicted glycemic control. Moderation was not present, as the numeric health literacy $\mathrm{X}$ responsibility interaction term was not significant, $b=0.00, p=.69,95 \% \mathrm{CI}[-0.03$, 0.02].

The overall model with family conflict moderating numeric health literacy and glycemic control was not significant, $F(3,61)=1.13, p=.34, R^{2}=.05$ (see Table 8$)$. Numeric health literacy did not significantly predict glycemic control, $b=-0.25, p=.30,95 \%$ CI [-0.73, 0.23] Family conflict was not a significant predictor of glycemic control, $b=-0.03, p=.65,95 \%$ CI [$0.18,0.11]$. The numeric health literacy $\mathrm{X}$ family conflict interaction term did not significantly predict glycemic control, $b=0.00, p=.62,95 \% \mathrm{CI}[-0.01,0.02]$; therefore, moderation was not present.

The model with parental supportive behavior as a potential moderator of numeric health literacy and glycemic control was not significant, $F(3,61)=1.91, p=.14, R^{2}=.09$ (see Table 8). Neither numeric health literacy, $b=0.00, p=.99,95 \%$ CI [-0.63, 0.63], nor supportive behavior, $b=0.00, p=.97,95 \%$ CI [-0.22, 0.23], were significant predictors of glycemic control. Supportive behavior was not a moderator, as the numeric health literacy X parental supportive behavior interaction term did not significantly predict glycemic control, $b=0.00, p=.66,95 \%$ CI $[-0.02,0.02]$.

The overall model with numeric health literacy and glycemic control, with parental nonsupportive behavior as a hypothesized moderator, was significant, $F(3,61)=3.94, p=.01, R^{2}=$ .16 (see Table 8). Numeric health literacy did not significantly predict glycemic control, $b=$ $0.29, p=.17,95 \%$ CI $[-0.13,0.71]$. Parental non-supportive behavior was a significant predictor 
of glycemic control, $b=0.30, p=.01,95 \% \mathrm{CI}[0.08,0.53]$; however, the numeric health literacy $\mathrm{X}$ non-supportive interaction was not significant, $b=-0.02, p=.08,95 \%$ CI [-0.04, 0.00], indicating moderation was not present.

\section{Discussion}

T1DM is a medical condition requiring a complex regimen to achieve good control of blood glucose levels. Little is known about which variables contribute to successful transition from pediatric to adult care, as it is thought that multiple domains are involved. It was hypothesized that health literacy and family factors may be relevant when considering transition in this population. The aims of this study were to: 1) examine the association of adolescent health literacy with transition readiness and with health outcome (i.e., glycemic control) in adolescents with T1DM; and 2) explore the extent to which family factors (i.e., diabetes management responsibility, family conflict, parental supportive behavior) served as moderators in adolescent health literacy predicting transition readiness and health outcome in T1DM (i.e., glycemic control). To address these aims, 70 adolescents with T1DM and their parents were recruited from a pediatric endocrinology clinic, with 65 adequately completing a series of measures assessing these constructs.

Results from this sample revealed adolescent T1DM knowledge was moderately associated with measures of written and numerical health literacy. This is consistent with the health literacy framework proposed by the Institute of Medicine (US) Committee on Health Literacy (2004), which demonstrated that knowledge and health literacy are related, yet distinct, constructs. According to this model, conceptual knowledge is one component of health literacy, along with print literacy (i.e., writing, reading), numeracy, and oral literacy (i.e., listening, speaking). In the current study, the DKT2 was selected to assess conceptual knowledge of T1DM 
and appropriate management. The diabetes-specific questionnaire chosen to measure numeric health literacy, the DNT-14, builds upon this knowledge, requiring participants to utilize this conceptual health knowledge to demonstrate capability of completing diabetes-related calculations. Although not diabetes-specific, the TOFHLA-R was selected to assess health-related written ability in a similar manner. Again, this measure required participants to demonstrate an ability to use their reading and writing knowledge to work with written health information effectively. Given the correlation among diabetes knowledge and the measures of health literacy, it is possible that knowledge accounted for greater variance than health literacy in the models, as noted in the results presented in the Appendix.

Contrary to hypotheses supported by the literature in adolescents with T1DM and parents of youth with T1DM (e.g., Mulvaney et al., 2013; Pulgarón et al., 2014), health literacy was not significantly associated with transition readiness or glycemic control. Furthermore, family factors (i.e., T1DM management responsibility, family conflict, parental supportive and nonsupportive behavior) did not serve as moderators in health literacy predicting transition readiness and glycemic control. Generally, these data contradict the results of studies with similar T1DM samples that demonstrated a significant association among adolescent numeric health literacy, using the DNT-14, and important outcomes, including glycemic control (Mulvaney et al., 2013). Yet, descriptive statistics of the DNT-14 and HbA1c values reported in these samples were similar to those of the current study. However, general diabetes knowledge was not included as a variable in this validation study (Mulvaney et al., 2013), as it was in the current study.

Research with other pediatric populations, such as those with human immunodeficiency virus (HIV), has also yielded varied results between health literacy and adherence-related outcomes. For example, greater health literacy predicted higher medication adherence and lower 
viral load for adults, but not for adolescents (Murphy et al., 2010; Navarra, Neu, Toussi, Nelson, \& Larson, 2014). Murphy and colleagues (2010) hypothesized this inconsistency may have resulted from other factors affecting adherence, including the comprehensive care provided at the health care center. Indeed, Navarra and colleagues (2014) noted health beliefs (e.g., expecting positive outcomes from taking medication) were more predictive of adherence than health literacy. Given the complexity of the T1DM regimen and the multiple daily treatment tasks prescribed, it is possible that other variables related to implementing these health literacy skills (e.g., self-efficacy, health beliefs) may be more relevant to adherence in adolescent T1DM (Herge et al., 2012). Research indicates there are a variety of constructs important to consider for both transition readiness and health literacy. As noted previously, there is evidence that preexisting factors (e.g., sociodemographic, access to healthcare, neurocognitive functioning), modifiable variables (e.g., knowledge, skills, motivation, relationships, communication), readiness to transition, and influence of systems all interact to impact health, (e.g., A1c), behavioral (e.g., adherence), and emotional outcomes (Pierce \& Wysocki, 2015). Therefore, it is possible that these numerous other patient characteristics or behaviors are more relevant to transition readiness and glycemic control than health literacy and family factors.

Significant correlations among variables in this study indicated other factors may be pertinent to a model of health literacy and transition readiness in this population. Notably, greater written health literacy was moderately associated with greater parental responsibility. This is consistent with Manganello's (2008) health literacy framework, which noted that caregivers may directly influence their adolescent's health literacy. For instance, research indicates more frequent parental reading and encouragement of reading at home was associated with increased adolescent literacy and interest in learning (Manganello, 2008; Strommen \& Mates, 2004). 
Although this research was conducted in general literacy, these findings may extend to health literacy. As the correlation from the current study suggest, caregivers who take more responsibility for T1DM management may engage in more modeling and encouragement of written health literacy in their adolescents. Greater written health literacy was also significantly associated with less family conflict. T1DM-specific family conflict has previously been associated with greater perceived parental burden (Hood et al., 2007). Although a causal relation is unable to be determined in the current study, it may be possible that more adolescent competency in written health literacy may decrease the perceived burden on caregivers, reducing the likelihood of conflict. In general, greater parental responsibility has been associated with better health outcomes (e.g., more frequent blood glucose monitoring; Vesco et al., 2010). The significant association among greater parental responsibility and greater transition readiness observed in this study was consistent with previous research reinforcing the importance of parental support in T1DM management. Notably, higher parental responsibility was significantly related to fewer parental supportive behaviors in this sample. The measure of parent responsibility used in the current study accounted for adolescents' perceptions of both direct (e.g., remembering times when blood sugar should be monitored) and indirect (e.g., remembering day of clinic appointments) T1DM management tasks. The measure assessing perceived parental supportive behaviors generally asked about support related to direct management tasks (e.g., praising for following their prescribed diet). There may be fewer opportunities for caregivers to engage in supportive behaviors when the caregivers already have primary responsibility for the tasks themselves. Finally, parental supportive and non-supportive behaviors were significantly positive associated. Although this contradicts previous research that 
found no relation (Lewin et al., 2005), it may be possible that this sample demonstrated greater parental engagement in adolescent care across supportive and non-supportive behaviors.

Overall, this study has multiple strengths which contribute to the existing literature. Namely, caregiver health literacy has been evaluated across pediatric populations and in T1DM. However, the current study's focus on adolescent health literacy is novel, as no study to date has highlighted the role of multiple measures of adolescent health literacy in T1DM, especially during the important developmental time period before transitioning to adult care. Additionally, T1DM-specific measures were used when available. This was particularly important for the measures of health literacy (i.e., numeracy) and knowledge, which are domain-specific constructs. All measures of family factors utilized in this study also were specific to T1DM, which provide a more detailed description of family functioning in this population. Additionally, there was good variability among HbA1c values to represent patients with different levels of glycemic control. Similarly, there was good variability among some demographic variables (e.g., caregiver education, income), making it more likely that this sample represents the greater population of adolescents with T1DM and their families.

The results of the current study should be interpreted in the context of multiple limitations. The research was conducted within the constraints of a busy interdisciplinary clinic setting with limited physical space. As a result, adolescents frequently completed the measures in the presence of at least one caregiver. Study staff discovered a limited number of participants were likely receiving assistance from a caregiver, although this may have happened more frequently than was observed. Notably, these participants' scores did not differ significantly from the overall sample, and current descriptive results (i.e., means, standard deviations) were 
consistent with those of other samples reported in the literature (e.g., Fitzgerald et al., 2016; Mulvaney et al., 2013). Nonetheless, this phenomenon may have influenced study results. Additionally, due to the absence of T1DM-specific measures of health literacy in the literature, print literacy and numeracy were combined for analyses in this study. Notably, exploratory analyses separating these two health literacy measures in the moderation analyses resulted in non-significant findings as well. It is possible that these measures did not fully or adequately evaluate all components of health literacy in this study; however, there is no one measure that adequately accounts for all proposed elements of health literacy in adolescents. The current research is consistent with other studies which evaluate one rather than multiple components of health literacy. Health literacy is a complex topic; therefore, constructs hypothesized to be most relevant (i.e., numeracy, written) were selected for this current study. Validated measures of health literacy in adolescents are sparse, and measures tend to assess one conceptual component of health literacy, frequently written health literacy. Moreover, validated diabetes-specific health literacy measures are limited, with the DNT-14 as the only published one to date. In the absence of other diabetes-specific health literacy measures, a written measure commonly used in other pediatric studies (TOFHLA-R) was selected. Given the lack of a validated measure of overall health literacy in this population, future research may consider how other components of health literacy, such as oral literacy, may account for these outcomes.

Another limitation in the current study was the reliance on the adolescents' ratings for most variables. Although this was a unique contribution to the literature, including other raters (e.g., caregiver, medical provider) would likely provide a more comprehensive description and may elicit different results. For instance, the measures assessing family variables and transition readiness rely on the adolescents' report. As a result, these responses may not have objectively 
represented the health behaviors in which adolescents engage or accurately reflect the family's interactions. Allowing the caregivers or medical providers to include their perspectives may have yielded different results.

Importantly, the racial and ethnic variability in this sample was limited and not representative of the greater T1DM population, despite being representative of the geographic region used for data collection. Furthermore, as is the case in all voluntary studies, self-selection bias may have resulted in differences between our study sample and families who did not participate, especially due to the novelty of psychosocial data collection in this clinic. For instance, families with greater health literacy, better family functioning, and/or greater glycemic control may have been more likely to participate. Moreover, this study was cross-sectional, therefore only associations and not causal relations can be inferred.

There are some future directions that may have the potential to address these limitations. To decrease the potential for patient-caregiver collaboration on measures that may influence results, efforts could be made to separate participants after consenting when completing study measures (e.g., in another clinic room or a private area of waiting room). Measures of other components of health literacy may provide more insight into the role of health literacy in the outcomes. For instance, oral literacy (e.g., listening comprehension, speaking) may be an important element that was not assessed in the current study. A future study could include other measures of health literacy to objectively account for oral health literacy. For example, the Health Literacy Assessment Scale for Adolescents (HAS-A) is a self-report scale that includes a subscale rating regarding the extent to which adolescents communicate about healthcare needs (Manganello et al., 2015). This measure asks adolescents about their perception of their skills related to health literacy but is not an objective measure of the extent to which they engage in 
these tasks. Furthermore, responses on the communication subscale are likely to be influenced by provider traits rather than purely reflecting adolescents' oral health literacy skills (Manganello et al., 2015). Alternatively, the Rapid Estimate of Adolescent Literacy in Medicine (REALM-Teen) is a screening tool involving the pronunciation of health-related words to assess health literacy and reading level (Davis et al., 2006). This measure would likely have provided more information about adolescent speaking ability (i.e., pronunciation); however, this measure would not have provided more comprehensive information about how adolescents communicate about or understand their health needs.

The use of the $T R A Q$ to assess transition readiness may have limited our conclusions about the complete transition process from pediatric to adult care. The $T R A Q$ is the most widely used transition measure in the pediatric literature and has the best psychometric properties among other measures of transition (Zhang et al., 2014). Its strengths include its development, which utilizing theory from multiple fields and stakeholders (Schwartz et al., 2014).

Nevertheless, it is not yet known the extent to which the TRAQ, or other pediatric transition measures reviewed by Schwartz and colleagues (2014), have predictive validity associating scores with patient transition outcomes. Furthermore, the item response options are based on research regarding behavior change, ranging from "No, I do not know how" to "Yes, I always do this when I need to." However, there is no choice for adolescents to respond that they know how to perform a task but do not do so. Given the importance of motivation and health beliefs to outcomes in adolescent T1DM (Pierce \& Wysocki, 2015), this appears to be a limitation of the $T R A Q$. Consequently, future research should examine motivation and self-efficacy as it relates to health literacy and transition. 
Future analyses may also include caregiver and provider ratings of constructs (e.g., transition readiness, family responsibility) to provide more information as well as potential discrepancies in reporting on adolescent and family functioning. Moreover, a more racially and ethnically diverse sample could be obtained from the addition of more sites and would likely lead to more statistically robust results to provide a more representative description of the T1DM population. Overall, the proper management of T1DM requires a complex treatment regimen, and there are multiple psychosocial factors to consider in the transition from pediatric to adult care as well as adherence. These future directions may lead to a more comprehensive and representative evaluation of health literacy, family factors, and outcomes for adolescents with T1DM and their families. Identifying and targeting these modifiable variables through brief intervention may lead to improved clinical outcomes, specifically regarding transition readiness and adherence. 


\section{References}

American Diabetes Association. (2019a). Food \& fitness. Retrieved on July 10, 2019 from http://www.diabetes. org/food-and-fitness/

American Diabetes Association. (2019b). Insulin \& other injectables. Retrieved on July 10, 2019 from http://www.diabetes.org/living-with-diabetes/treatment-and-care/medication/ insulin/

American Diabetes Association. (2019c). Standards of medical care in diabetes-2019. Diabetes Care, 42(Suppl. 1), S1-S193. doi.org/10.2337/dc19-S013

Anderson, B. J., Auslander, W. F., Jung, K. C., Miller, J. P., \& Santiago, J. V. (1990). Assessing family sharing of diabetes responsibilities. Journal of Pediatric Psychology, 15, 477-492.

Anderson, B. J., Vangsness, L., Connell, A., Butler, D., Goebel-Fabbri, A., \& Laffel, L. M. B. (2002). Family conflict, adherence, and glycaemic control in youth with short duration type 1 diabetes. Diabetic Medicine, 19, 635-642.

Arnett, J. J. (2000). Emerging adulthood: A theory of development from the late teens through the twenties. American Psychologist, 55, 469-480. doi: 10.1037/0003-066X.55.5.469

Ball, G. D., Huang, T. T. K., Gower, B. A., Cruz, M. L., Shaibi, G. Q., Weigensberg, M. J., \& Goran, M. I. (2006). Longitudinal changes in insulin sensitivity, insulin secretion, and $\beta$ cell function during puberty. The Journal of Pediatrics, 148, 16-22. doi:10.1016/j.jpeds. 2005.08.059

Brown, S. L., Teufel, J. A., \& Birch, D. A. (2007). Early adolescents perceptions of health and health literacy. Journal of School Health, 77, 7-15. doi:10.1111/j.17461561.2007.00156.x 
Bryden, K. S., Peveler, R. C., Stein, A., Neil, A., Mayou, R. A., \& Dunger, D. B. (2001). Clinical and psychological course of diabetes from adolescence to young adulthood: A longitudinal cohort study. Diabetes Care, 24, 1536-1540. doi:10.2337/ diacare.24.9.1536

Cavanaugh, K., Huizinga, M. M., Wallston, K. A., Gebretsadik, T., Shintani, A., Davis, D., .. . Rothman, R. L. (2008). Association of numeracy and diabetes control. Annals of Internal Medicine, 148, 737-746.

Centers for Disease Control and Prevention. (2014). National diabetes statistics report: Estimates of diabetes and its burden in the United States, 2014. Atlanta, GA: U.S. Department of Health and Human Services.

Centers for Disease Control and Prevention. (2017). National diabetes statistics report: Estimates of diabetes and its burden in the United States, 2017. Atlanta, GA: U.S. Department of Health and Human Services.

Chisolm, D. J., \& Buchanan, L. (2007). Measuring adolescent functional health literacy: A pilot validation of the Test of Functional Health Literacy in Adults. Journal of Adolescent Health, 41, 312-314. doi:10.1016/j.jadohealth.2007.04.015

Dabelea, D., Mayer-Davis, E. J., Saydah, S., Imperatore, G., Linder, B., Divers, J., . . Hamman, R. F. (2014). Prevalence of type 1 and type 2 diabetes among children and adolescents from 2001 to 2009. JAMA, 311, 1778-1786. doi:10.1001/jama.2014.3201

Davis, T.C., Wolf, M.S., Arnold, C. L., Byrd, R. S., Long, S.W., Springer, T., . . Bocchini, J.A. (2006). Development and validation of the Rapid Estimate of Adolescent Literacy in Medicine (REALM-Teen): A tool to screen adolescents for below-grade reading in health care settings. Pediatrics, 118, e1707-1714. doi: 10.1542/peds.2006-1139 
DeWalt, D. A., \& Hink, A. (2009). Health literacy and child health outcomes: A systematic review of the literature. Pediatrics, 124, S265-S274. doi:10.1542/peds.2009-1162B

Fitzgerald, J. T., Funnell, M. M., Anderson, R. M., Nwankwo, R., Stansfield, R. B., \& Piatt, G. A. (2016). Validation of the Revised Brief Diabetes Knowledge Test (DKT2). The Diabetes Educator, 42, 178-187. doi: 10.1177/0145721715624968

Follansbee, D. S. (1989). Assuming responsibility for diabetes management: What age? What price? The Diabetes Educator, 15, 347-353. doi:10.1177/014572178901500417

Ghaddar, S. F., Valerio, M. A., Garcia, C. M., \& Hansen, L. (2012). Adolescent health literacy: The importance of credible sources for online health information. Journal of School Health, 82, 28-36. doi:10.1111/j.1746-1561.2011.00664.x

Gray, N. J., Klein, J. D., Noyce, P. R., Sesselberg, T. S., \& Cantrill, J. A. (2005). The Internet: A window on adolescent health literacy. Journal of Adolescent Health, 37, 243.e1-243.e7. doi:10.1016/j.jadohealth.2004.08.023

Hassan, K., \& Heptulla, R. A. (2010). Glycemic control in pediatric type 1 diabetes: Role of caregiver literacy. Pediatrics, 125, e1104-e1108. doi:10.1542/peds.2009-1486

Herge, W. M., Streisand, R., Chen, R., Holmes, C., Kumar, A., \& Mackey, E. R. (2012). Family and youth factors associated with health beliefs and health outcomes in youth with type 1 diabetes. Journal of Pediatric Psychology, 37, 980-989. doi: 10.1093/jpepsy/jss067

Hood, K. K., Butler, D. A., Anderson, B. J., \& Laffel, L. M. B. (2007). Updated and revised Diabetes Family Conflict Scale. Diabetes Care, 30, 1764-1769. doi:10.2337/dc06-2358

Huizinga, M. M., Elasy, T. A., Wallston, K. A., Cavanaugh, K., Davis, D., Gregory, R. P., \& Rothman, R. L. (2008). Development and validation of the Diabetes Numeracy Test (DNT). BMC Health Services Research, 8. doi:10.1186/1472-6963-8-96 
Imperatore, G., Boyle, J. P., Thompson, T. J., Case, D., Dabelea, D., Hamman, R. F., . . .

Standiford, D. (2012). Projections of type 1 and type 2 diabetes burden in the U.S. population aged < 20 years through 2050. Diabetes Care, 35, 2515-2520. doi:10.2337/ dc12-0669

Ingerski, L. M., Anderson, B. J., Dolan, L. M., \& Hood, K. K. (2010). Blood glucose monitoring and glycemic control in adolescence: Contribution of diabetes-specific responsibility and family conflict. Journal of Adolescent Health, 47, 191-197. doi:10.1016/j.jadohealth. 2010.01.012

Ingersoll, G. M., Orr, D. P., Herrold, A. J., \& Golden, M. P. (1986). Cognitive maturity and selfmanagement among adolescent with insulin-dependent diabetes mellitus. The Journal of Pediatrics, 108, 620-623.

Insabella, G., Grey, M., Knafl, G., \& Tamborlane, W. (2007). The transition to young adulthood in youth with type 1 diabetes on intensive treatment. Pediatric Diabetes, 8, 228-234. doi: 10.1111/j.1399-5448.2007.00266.x

Institute of Medicine (US) Committee on Health Literacy. (2004). Health literacy: A prescription to end confusion. L. Nielsen-Bohlman, A. M. Panzer, \& D. A. Kindig, Eds. Washington, DC: National Academies Press.

Ivey, J. B., Wright, A., \& Dashiff, C. J. (2009). Finding the balance: Adolescents with type 1 diabetes and their parents. Journal of Pediatric Health Care, 23, 10-18. doi: :10.1016/j. pedhc.2007.12.008

Jacobson, A. M., Hauser, S. T., Willett, J. B., Wolfsdorf, J. I., Dvorak, R., Herman, L., \& De Groot, M. (1997). Psychological adjustment to IDDM: 10-year follow-up of an onset cohort of child and adolescent patients. Diabetes Care, 20, 811-818. 
Janisse, H. C., Naar-King, S., \& Ellis, D. (2010). Brief report: Parent's health literacy among high-risk adolescents with insulin dependent diabetes. Journal of Pediatric Psychology, 35, 436-440. doi:10.1093/jpepsy/jsp077

Jeffcoate, S. L. (2004). Diabetes control and complications: The role of glycated haemoglobin, 25 years on. Diabetic Medicine, 21, 657-665. doi:10.1046/j.1464-5491.2003.01065.x

Juvenile Diabetes Research Foundation. (2019). Type 1 diabetes facts. Retrieved July 10, 2019 from http://www.jdrf.org/about/fact-sheets/type-1-diabetes-facts/

Kadiyala, P., Walton, S., \& Sathyapalan, T. (2014). Insulin induced lipodystrophy. British Journal of Diabetes, 14, 131-133. doi:10.15277/bjdvd.2014.036

Laffel, L. M. B., Vangsness, L., Connell, A., Goebel-Fabbri, A., Butler, D., \& Anderson, B. J. (2003). Impact of ambulatory, family-focused teamwork intervention on glycemic control in youth with type 1 diabetes. The Journal of Pediatrics, 142, 409-416. doi:10.1067/mpd. 2003.138

Lancaster, B. M., Gadaire, D. M., Holman, K., \& LeBlanc, L. A. (2015). Association between diabetes treatment adherence and parent-child agreement regarding treatment responsibilities. Families, Systems, \& Health, 33, 120-125. http://dx.doi.org/10.1037/ fsh0000092

Leonard, B. J., Garwick, A., \& Adwan, J. Z. (2005). Adolescents' perceptions of parental roles and involvement in diabetes management. Journal of Pediatric Nursing, 20, 405-414. doi:10.1016/j.pedn.2005.03.010

Lewin, A. B., Geffken, G. R., Heidgerken, A. D., Duke, D. C., Novoa, W., Williams, L. B., \& Storch, E. A. (2005). The Diabetes Family Behavior Checklist: A psychometric 
evaluation. Journal of Clinical Psychology in Medical Settings, 12, 315-322. doi:10. 1007/s10880-005-7817-X

Lewin, A. B., Heidgerken, A. D., Geddken, G. R., Williams, L. B., Storch, E. A., Gelfand, K. M., \& Silverstein, J. H. (2006). The relation between family factors and metabolic control: The role of diabetes adherence. Journal of Pediatric Psychology, 32, 174-183. doi:10.1093/jpepsy/jsj004

Livingstone, S. J., Levin, D., Looker, H. C., Lindsay, R.S., Wild, S. H., Joss, N., . . Scottish Renal Registry. (2015). Estimated life expectancy in a Scottish cohort with type 1 diabetes, 2008-2010. JAMA, 313, 37-44. doi: 10.1001/jama.2014.16425

Manganello, J. A. (2008). Health literacy and adolescents: A framework and agenda for future research. Health Education Research, 23, 840-847. doi:10.1093/her/cym069

Manganello, J.A., FeVellis, R. F., Davis, T. C., \& Schottler-Thal, C. (2015). Development of the Health Literacy Assessment Scale for Adolescents (HAS-A). Journal of Communication in Healthcare, 8, 172-184. doi: 10.1179/1753807615Y.0000000016

Mayer-Davis, E. J., Lawrence, J. M., Dabelea, D., Divers, J., Isom, S., Dolan, L., . . Wagenknecht, L. (2017). Incidence trends of type 1 and type 2 diabetes among youths, 2002-2012. New England Journal of Medicine, 376, 1419-1429. doi: 10.1056/ NEJMoa1610187

McKelvey, J., Waller, D. A., North, A. J., Marks, J. F., Schreiner, B., Travis, L. B., \& Murphy, J. N. (1993). Reliability and validity of the Diabetes Family Behavior Scale (DFBS). The Diabetes Educator, 19, 125-132. doi:10.1177/014572179301900206 
Miller-Johnson, S., Emery, R. E., Marvin, R. S., Clarke, W., Lovinger, R., \& Martin, M. (1994). Parent-child relationships and the management of insulin-dependent diabetes mellitus. Journal of Consulting and Clinical Psychology, 62, 603-610.

Monaghan, M., Hilliard, M., Sweenie, R., \& Riekert, K. (2013). Transition readiness in adolescents and emerging adults with diabetes: The role of patient-provider communication. Current Diabetes Reports, 13, 900-908. doi:10.1007/s11892-013-0420-x

Morris, A. D., Boyle, D. I., McMahon, A. D., Greene, S. A., MacDonald, T. M., \& Newton, R. W. (1997). Adherence to insulin treatment, glycaemic control, and ketoacidosis in insulin-dependent diabetes mellitus. The Lancet, 350, 1505-1510.

Mulvaney, S. A., Lilley, J. A., Cavanaugh, K. L., Pittel, E. J., \& Rothman, R. L. (2013). Validation of the Diabetes Numeracy Test with adolescents. Journal of Health Communication, 18, 795-804, doi:10.1080/10810730.2012.757394

Murphy, D. A., Lam, P., Naar-King, S., Harris, D. R., Parsons, J. T., \& Muenz, L. R. (2010). Health literacy and antiretroviral adherence among HIV-infected adolescents. Patient Education and Counseling, 79, 25-29. doi: 10.1016/j.pec.2009.07.014

Navarra, A. M., Neu, N., Toussi, S., Nelson, J., \& Larson, E. L. (2014). Health literacy and adherence to antiretroviral therapy among HIV-infected youth. Journal of the Association of Nurses in AIDS Care, 25, 203-213. doi: 10.1016/j.jana.2012.11.003

Nutbeam, D. (2009). Defining and measuring health literacy: What can we learn from literacy studies? International Journal of Public Health, 54, 303-305. doi:10.1007/s00038-0090050-X 
Parker, R. M., Baker, D. W., Williams, M. V., \& Nurss, J. R. (1995). The Test of Functional Health Literacy in Adults: A new instrument for measuring patients' literacy skills. Journal of General Internal Medicine, 10, 537-541.

Pettitt, D. J., Talton, J., Dabelea, D., Divers, J., Imperatore, G., Lawrence, J. M., . . Hamman, R. F. (2014). Prevalence of diabetes in U.S. youth in 2009: The SEARCH for Diabetes in Youth Study. Diabetes Care, 37, 402-408. doi:10.2337/dc13-1838

Pierce, J. S., \& Wysocki, T. (2015). Topical review: Advancing research on the transition to adult care for type 1 diabetes. Journal of Pediatric Psychology, 40, 1041-1047. doi:10. 1093/jpepsy/jsv064

Prochaska, J. O., \& DiClemente, C. C. (1986). Toward a comprehensive model of change. In W. R. Miller \& N. Heather (Eds.), Treating addictive behaviors (pp. 3-27). New York, NY: Plenum.

Pulgarón, E. R., \& Sanders, L. M. (2014). Glycemic control in young children with diabetes: The role of parental health literacy. Patient Education and Counseling, 94, 67-70. http://dx. doi.org/10.1016/j.pec.2013.09.002

Rubin, R., Young-Hyman, D., \& Peyrot, M. (1989). Parent-child responsibility and conflict in diabetes care. Diabetes, 38(Suppl. 2), 28.

Sanders, L. M., Federico, S., Klass, P., Abrams, M. A., \& Dreyer, B. (2009). Literacy and child health: A systematic review. Archives of Pediatric \& Adolescent Medicine, 163, 131-140. doi:10.1001/archpediatrics.2008.539

Sawicki, G. S., Lukens-Bull, K., Yin, X., Demars, N., Huang, I., Livingood, W., . . Wood, D. (2011). Measuring the transition readiness of youth with special healthcare needs: 
Validation of the TRAQ_-Transition Readiness Assessment Questionnaire. Journal of Pediatric Psychology, 36, 160-171. doi:10.1093/jpepsy/jsp128

Schilling, L. S., Knafl, K. A., \& Grey, M. (2006). Changing patterns of self-management in youth with type 1 diabetes. Journal of Pediatric Nursing, 21, 412-424. doi:10.1016/j. pedn.2006.01.034

Schwartz, L. A., Daniel, L. C., Brumley, L. D., Barakat, L. P., Wesley, K. M., \& Tuchman, L. K. (2014). Measures of readiness to transition to adult health care for youth with chronic physical health conditions: A systematic review and recommendations for measurement testing and development. Journal of Pediatric Psychology, 39, 588-601. doi: 10.1093/ jpepsy/jsu028

Strommen, L. T. \& Mates, B. F. (2004). Learning to love reading: Interviews with older children and teens. Journal of Adolescent and Adult Literacy, 48, 188-200. doi: 10.1598/JAAL.48. 3.1

Sullivan-Bolyai, S., Bova, C., Johnson, K., Cullen, K., Jaffarian, C., Quinn, D., . . Gupta, O. (2014). Engaging teens and parents in collaborative practice: Perspectives on diabetes self-management. The Diabetes Educator, 40, 178-190. doi:10.1177/0145721713520568

The Diabetes Control and Complications Trial Research Group. (1993). The effect of intensive treatment of diabetes on the development and progression of long-term complications in insulin-dependent diabetes mellitus. The New England Journal of Medicine, 329, 977986. doi:10.1056/NEJM199309303291401

U.S. Department of Health and Human Services. (2010). National action plan to improve health literacy. Washington, DC: Author. 
Vesco, A. T., Anderson, B. J., Laffel, L. M., Dolan, L. M., Ingerski, L. M., \& Hood, K. K. (2010). Responsibility sharing between adolescents with type 1 diabetes and their caregivers: Importance of adolescent perceptions on diabetes management and control. Journal of Pediatric Psychology, 35, 1168-1177. doi:10.1093/jpepsy/jsq038

Wood, D. L., Sawicki, G. S., Miller, D., Smotherman, C., Lukens-Bull, K., Livingood, W. C., . . Kraemer, D. F. (2014). The Transition Readiness Assessment Questionnaire (TRAQ): Its factor structure, reliability, and validity. Academic Pediatrics, 14, 415-422. doi:10. 1016/j.acap.2014.03.008

Wysocki, T. (1993). Associations among teen-parent relationships, metabolic control, and adjustment to diabetes in adolescents. Journal of Pediatric Psychology, 18, 441-452.

Wysocki, T., Greco, P., Harris, M. A., Bubb, J., \& White, N. H. (2001). Behavior therapy for families of adolescents with diabetes: Maintenance of treatment effects. Diabetes Care, 24, 441-446.

Wysocki, T., Harris, M. A., Buckloh, L. M., Mertlich, D., Lochrie, A. S., Mauras, N., \& White, N. H. (2007). Randomized trial of Behavioral Family Systems Therapy for Diabetes: Maintenance of effects on diabetes outcomes in adolescents. Diabetes Care, 30, 555-560. doi:10.2337/dc06-1613

Wysocki, T., Harris, M. A., Greco, P., Bubb, J., Danda, C. E., Harvey, L. M., . . White, N. H. (2000). Randomized, controlled trial of behavior therapy for families of adolescents with insulin-dependent diabetes mellitus. Journal of Pediatric Psychology, 25, 23-33.

Wysocki, T., Taylor, A., Hough, B. S., Linscheid, T. R., Yeates, K. O. \& Naglieri, J. A. (1996). Deviation from developmentally appropriate self-care autonomy: Association with diabetes outcomes. Diabetes Care, 19, 119-125. 
Yin, H. S., Dreyer, B. P., van Schaick, L., Foltin, G. L., \& Mendelsohn, A. L. (2008).

Randomized controlled trial of a pictogram-based intervention to reduce liquid medication dosing errors and improve adherence among caregivers of young children. Archives of Pediatric \& Adolescent Medicine, 162, 814-822. doi:10.1001/archpedi.162.9. 814

Yin, H. S., Johnson, M., Mendelsohn, A. L., Abrams, M. A., Sanders, L. M., \& Dreyer, B. P. (2009). The health literacy of parents in the United States: A nationally representative study. Pediatrics, 124, S289-S298. doi:10.1542/peds.2009-1162E

Zhang, L. F., Ho, J. S. W., \& Kennedy, S. E. (2014). A systematic review of the psychometric properties of transition readiness assessment tools in adolescents with chronic disease. BMC Pediatrics, 14. doi:10.1186/1471-2431-14-4 
Table 1

Participant Characteristics

\begin{tabular}{lc}
\hline \multicolumn{1}{c}{ Variable $(\boldsymbol{N}=\mathbf{6 5})$} & $\boldsymbol{n}(\boldsymbol{\%})$ \\
\hline Patient gender & \\
Male & $36(55 \%)$ \\
Female & $29(45 \%)$ \\
Patient race & \\
White & $62(95 \%)$ \\
American Indian/Alaskan Native & $1(1 \%)$ \\
Black/African American & $1(1 \%)$ \\
Multiracial & $1(1 \%)$ \\
Patient ethnicity & \\
Not Hispanic/Latino & $65(100 \%)$ \\
Caregiver relationship & \\
Mother & \\
Father & $55(85 \%)$ \\
Grandmother & $7(11 \%)$ \\
Not reported & $2(3 \%)$ \\
Family structure & $1(1 \%)$ \\
Married to biological parent & \\
Single & $37(57 \%)$ \\
Blended & $19(29 \%)$ \\
Not reported & $7(11 \%)$ \\
Highest caregiver education level & $2(3 \%)$ \\
High school & \\
Some college/vocational training & $10(15 \%)$ \\
Bachelor's degree & $26(40 \%)$ \\
Master’s degree/doctoral degree & $17(26 \%)$ \\
Total family income & $12(19 \%)$ \\
Less than $\$ 10,000$ & \\
$\$ 10,000-14,999$ & $2(3 \%)$ \\
$\$ 15,000-24,999$ & $2(3 \%)$ \\
$\$ 25,000-34,999$ & $5(8 \%)$ \\
$\$ 35,000-49,999$ & $9(14 \%)$ \\
$\$ 50,000-74,999$ & $5(8 \%)$ \\
$\$ 75,000-99,999$ & $6(9 \%)$ \\
$\$ 100,000-149,999$ & $10(15 \%)$ \\
$\$ 150,000$ or greater & $12(19 \%)$ \\
Not reported & $9(14 \%)$ \\
& $5(8 \%)$ \\
\hline
\end{tabular}




\begin{tabular}{lc}
\hline \multicolumn{1}{c}{ Variable $(\boldsymbol{N = 6 5 )}$} & $\boldsymbol{n}(\boldsymbol{\%})$ \\
\hline Difficulty paying bills & $3(5 \%)$ \\
Always & $2(3 \%)$ \\
Most of the time & $7(11 \%)$ \\
Sometimes & $18(28 \%)$ \\
Rarely & $31(48 \%)$ \\
Never & $4(6 \%)$ \\
Not reported & \\
Insurance & \\
Private & $37(57 \%)$ \\
Public & $19(29 \%)$ \\
Not reported & $9(14 \%)$ \\
Treatment & \\
Insulin pump & $36(55 \%)$ \\
Multiple daily insulin injections & $30(46 \%)$ \\
Basal/bolus insulin injections & $7(11 \%)$ \\
Sources of health information & \\
Medical professionals & $54(83 \%)$ \\
Family who do not have T1DMM & $25(39 \%)$ \\
Websites & $23(35 \%)$ \\
Family who have T1DMM & $22(34 \%)$ \\
Friends who have T1DMM & $17(26 \%)$ \\
Television & $11(17 \%)$ \\
Print media & $10(15 \%)$ \\
Social media & $10(15 \%)$ \\
Friends who do not have T1DMM & $9(14 \%)$ \\
Advertisements & $6(9 \%)$ \\
Radio & $6(9 \%)$ \\
Other & $2(3 \%)$ \\
\hline
\end{tabular}

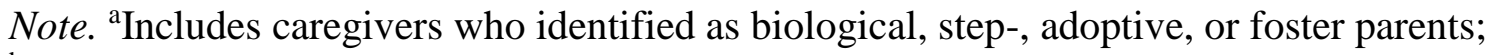

${ }^{\mathrm{b}}$ Blended = remarried to step-parent; Single = never been married, divorced, widowed, or living with partner 
Table 2

Primary Variable Raw Score Descriptives

\begin{tabular}{lcccc}
\hline \multicolumn{1}{c}{ Variable } & $\boldsymbol{M}$ & $\boldsymbol{S D}$ & Range & $\boldsymbol{\alpha} / \mathbf{K R 2 0}$ \\
\hline$D K T 2$ & 76.04 & 12.96 & $30-100$ & .66 \\
$D N T-14$ & 75.86 & 21.57 & $14-100$ & .80 \\
TOFHLA-R & 43.69 & 5.06 & $19-50$ & .84 \\
DFR $Q$ & 37.14 & 6.19 & $17-50$ & .89 \\
DFCS-R & 30.03 & 10.96 & $19-57$ & .96 \\
DFBC-C $(S)$ & 29.41 & 7.83 & $9-45$ & .78 \\
DFBC-C $(N-S)$ & 18.74 & 6.52 & $7-34$ & .72 \\
TRA $Q$ & 68.28 & 16.38 & $37-100$ & .92 \\
HbA1c & 8.70 & 1.84 & $5.7-14.4$ & --- \\
\hline
\end{tabular}

Note. DKT2 (Revised Brief Diabetes Test 2): higher scores = greater diabetes knowledge; DNT14 (Diabetes Numeracy Test-14): higher scores = greater diabetes-specific numeracy; TOFHLA$R$ (Test of Functional Health Literacy in Adults-Reading): higher scores = greater written health literacy; DFRQ (Diabetes Family Responsibility Questionnaire): higher scores = higher parental responsibility for diabetes management; DFCS-R (Diabetes Family Conflict Scale-Revised): higher scores = higher diabetes-related conflict; $D F B C-C(S)$ (Diabetes Family Behavior Checklist-Child Report, Supportive subscale): higher scores = greater frequency of parental supportive diabetes-related behaviors; DFBC-C $(N-S)$ (Diabetes Family Behavior ChecklistChild Report, Non-Supportive subscale): higher scores = greater frequency of parental nonsupportive diabetes-related behaviors; TRAQ (Transition Readiness Assessment Questionnaire): higher scores $=$ greater transition readiness; HbA1c: higher values = poorer glycemic control $(<$ 7.5\% recommended; ADA, 2017). 
Table 3

Primary Variable Correlations

\begin{tabular}{|c|c|c|c|c|c|c|c|c|c|}
\hline & $D K T 2$ & DNT-14 & TOFHLA-R & $D F R Q$ & $D F C S-R$ & $D F B C-C(S)$ & $D F B C-C(N-S)$ & $T R A Q$ & $\mathrm{HbA1c}$ \\
\hline DKT2 & --- & & & & & & & & \\
\hline TOFHLA-R & $0.44 * *$ & $0.43 * *$ & --- & & & & & & \\
\hline$D F C S-R$ & -0.22 & -0.21 & $-0.27 *$ & -0.22 & --- & & & & \\
\hline$D F B C-C(S)$ & -0.17 & -0.01 & -0.12 & $-0.30 *$ & 0.14 & --- & & & \\
\hline$D F B C-C(N-S)$ & -0.11 & 0.08 & -0.02 & -0.18 & 0.22 & $0.33 * *$ & --- & & \\
\hline
\end{tabular}

Note. DKT2: Revised Brief Diabetes Test 2; DNT-14: Diabetes Numeracy Test-14; TOFHLA-R: Test of Functional Health Literacy in Adults-Reading; DFRQ: Diabetes Family Responsibility Questionnaire; DFCS-R: Diabetes Family Conflict Scale-Revised; DFBC-C (S): Diabetes Family Behavior Checklist-Child Report, Supportive subscale; DFBC-C (N-S): Diabetes Family Behavior ChecklistChild Report, Non-Supportive subscale; TRAQ: Transition Readiness Assessment Questionnaire; HbA1c: glycemic control.

$* p<.05, * * p<.01$ 
Table 4

Demographic Variable Correlations with Primary Variables

\begin{tabular}{lcccccc}
\hline & Age & $\begin{array}{c}\text { Diagnosis } \\
\text { duration }\end{array}$ & Sex & $\begin{array}{c}\text { Caregiver } \\
\text { education }\end{array}$ & Income & Insurance \\
\hline DKT2 & -0.01 & -0.01 & 0.00 & 0.00 & 0.00 & 0.25 \\
DNT-14 & 0.13 & 0.02 & -0.03 & $0.25^{*}$ & 0.20 & 0.13 \\
TOFHLA-R & 0.18 & 0.03 & -0.02 & 0.11 & -0.13 & -0.02 \\
DFRQ & $0.31 *$ & 0.18 & 0.24 & -0.04 & -0.18 & -0.21 \\
DFCS-R & -0.05 & -0.22 & -0.04 & -0.15 & 0.02 & -0.16 \\
DFBC-C $(S)$ & 0.03 & -0.08 & -0.02 & 0.14 & 0.05 & -0.23 \\
DFBC-C $(N-S)$ & 0.01 & -0.05 & 0.09 & -0.02 & -0.07 & $-0.34^{*}$ \\
TRAQ & $0.42^{* *}$ & 0.16 & 0.19 & -0.05 & 0.06 & -0.06 \\
HbA1c & -0.03 & 0.12 & -0.12 & -0.21 & $-0.30 *$ & $-0.37^{* *}$ \\
\hline
\end{tabular}

Note. DKT2: Revised Brief Diabetes Test 2; DNT-14: Diabetes Numeracy Test-14; TOFHLA-R: Test of Functional Health Literacy in Adults-Reading; DFRQ: Diabetes Family Responsibility Questionnaire; DFCS-R: Diabetes Family Conflict Scale-Revised; DFBC-C (S): Diabetes Family Behavior Checklist-Child Report, Supportive subscale; DFBC-C $(N-S)$ : Diabetes Family Behavior Checklist-Child Report, Non-Supportive subscale; TRAQ: Transition Readiness Assessment Questionnaire; HbA1c: glycemic control; Age reported in years; Diagnosis duration reported in years; Sex: Male $=0$, Female $=1$; Caregiver education reported in the following categories: < \$10,000, \$10,000-\$14,999, \$15,000-\$24,999, \$25,000-\$34,999, \$35,000-\$49,000, $\$ 50,000-\$ 74,999, \$ 75,000-\$ 99,999, \$ 100,000-\$ 149,999, \geq \$ 150,000 ;$ Insurance: $0=$ Public, $1=$ Private.

$* p<.05, * * p<.01$ 
Table 5

Moderation Effect of Family Factors on the Relation between Written Health Literacy and Transition Readiness

\begin{tabular}{lccc}
\hline Predictor & $b$ & $p$ & $95 \%$ CI \\
\hline Written health literacy & -4.22 & .18 & $-10.46,2.03$ \\
Family responsibility & -2.81 & .46 & $-10.31,4.68$ \\
Written health literacy X Family responsibility & 0.09 & .30 & $-0.08,0.26$ \\
& & & \\
Written health literacy & 2.19 & .21 & $-1.23,5.62$ \\
Family conflict & 3.44 & .11 & $-0.76,7.65$ \\
Written health literacy X Family conflict & -0.08 & .09 & $-0.18,0.01$ \\
& & & \\
Written health literacy & 0.36 & .86 & $-3.62,4.34$ \\
Parental supportive behavior & 1.43 & .64 & $-4.33,7.02$ \\
Written health literacy X Supportive behavior & -0.03 & .69 & $-0.16,0.11$ \\
& & & \\
Written health literacy & 2.91 & .12 & $-0.75,6.56$ \\
Parental non-supportive behavior & 7.95 & .05 & $-0.10,16.00$ \\
Written health literacy X Non-supportive behavior & -0.18 & .06 & $-0.36,0.01$ \\
\hline
\end{tabular}


Table 6

Moderation Effect of Family Factors on the Relation between Written Health Literacy and Glycemic Control

\begin{tabular}{lccc}
\hline Predictor & $b$ & $p$ & $95 \%$ CI \\
\hline Written health literacy & -0.09 & .81 & $-0.85,0.67$ \\
Family responsibility & -0.04 & .94 & $-0.95,0.88$ \\
Written health literacy X Family responsibility & 0.00 & .89 & $-0.02,0.02$ \\
& & & \\
Written health literacy & -0.26 & .19 & $-0.65,0.13$ \\
Family conflict & -0.30 & .21 & $-0.78,0.17$ \\
Written health literacy X Family conflict & 0.01 & .20 & $0.00,0.02$ \\
& & & \\
Written health literacy & 0.08 & .73 & $-0.37,0.52$ \\
Parental supportive behavior & 0.11 & .72 & $-0.52,0.74$ \\
Written health literacy X Supportive behavior & 0.00 & .62 & $-0.02,0.01$ \\
& & & \\
Written health literacy & & .95 & $-0.41,0.43$ \\
Parental non-supportive behavior & 0.01 & .80 & $-0.81,1.04$ \\
Written health literacy X Non-supportive behavior & 0.00 & .88 & $-0.02,0.02$ \\
\hline
\end{tabular}


Table 7

Moderation Effect of Family Factors on the Relation between Numeric Health Literacy and Transition Readiness

\begin{tabular}{lccc}
\hline Predictor & $b$ & $p$ & $95 \%$ CI \\
\hline Numeric health literacy & -5.78 & .15 & $-13.78,2.23$ \\
Family responsibility & -0.86 & .47 & $-3.22,1.51$ \\
Numeric health literacy X Family responsibility & 0.16 & .13 & $-0.05,0.37$ \\
& & & \\
Numeric health literacy & 2.37 & .28 & $-1.96,6.70$ \\
Family conflict & 0.53 & .44 & $-0.82,1.87$ \\
Numeric health literacy X Family conflict & -0.06 & .36 & $-0.18,0.07$ \\
& & & \\
Numeric health literacy & -1.49 & .61 & $-7.28,4.29$ \\
Parental supportive behavior & -0.51 & .62 & $-2.55,1.53$ \\
Numeric health literacy X Supportive behavior & 0.07 & .47 & $-0.12,0.25$ \\
& & & \\
Numeric health literacy & 2.45 & .23 & $-1.55,6.45$ \\
Parental non-supportive behavior & 1.40 & .20 & $-0.75,3.55$ \\
Numeric health literacy X Non-supportive behavior & -0.10 & .30 & $-0.30,0.09$ \\
\hline
\end{tabular}


Table 8

Moderation Effect of Family Factors on the Relation between Numeric Health Literacy and Glycemic Control

\begin{tabular}{lccc}
\hline Predictor & $b$ & $p$ & $95 \%$ CI \\
\hline Numeric health literacy & 0.03 & .94 & $-0.90,0.97$ \\
Family responsibility & 0.09 & .53 & $-0.19,0.36$ \\
Numeric health literacy X Family responsibility & 0.00 & .69 & $-0.03,0.02$ \\
& & & \\
Numeric health literacy & -0.25 & .30 & $-0.73,0.23$ \\
Family conflict & -0.03 & .65 & $-0.18,0.11$ \\
Numeric health literacy X Family conflict & 0.00 & .62 & $-0.01,0.02$ \\
& & & \\
Numeric health literacy & 0.00 & .99 & $-0.63,0.63$ \\
Parental supportive behavior & 0.00 & .97 & $-0.22,0.23$ \\
Numeric health literacy X Supportive behavior & 0.00 & .66 & $-0.02,0.02$ \\
& & & \\
Numeric health literacy & 0.29 & .17 & $-0.13,0.71$ \\
Parental non-supportive behavior & 0.30 & .01 & $0.08,0.53$ \\
Numeric health literacy X Non-supportive behavior & -0.02 & .08 & $-0.04,0.00$ \\
\hline
\end{tabular}




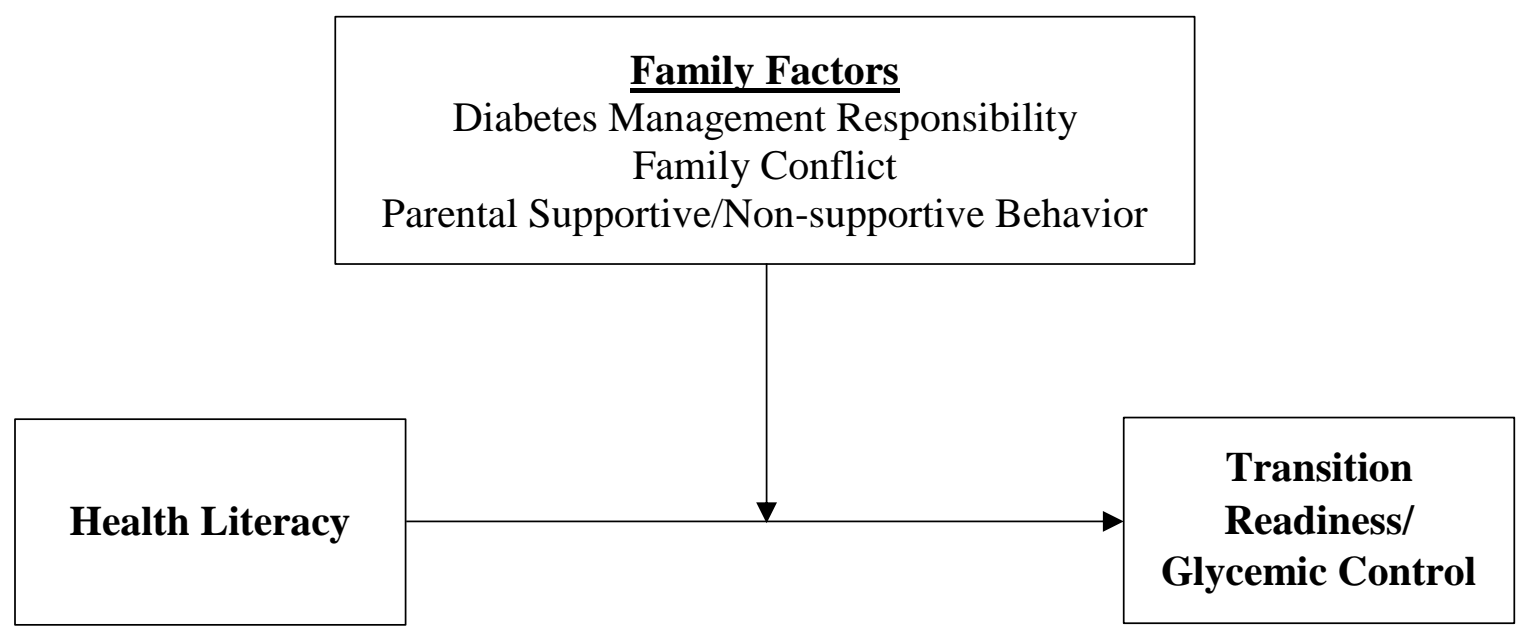

Figure 1. Proposed moderation model of family factors as moderators in adolescent health literacy predicting transition readiness and health outcome. 


\section{Appendix}

Moderation Effect of Family Factors on the Relation between Diabetes Knowledge, Written Health Literacy, and Transition Readiness

\begin{tabular}{lccc}
\hline Predictor & $b$ & $p$ & $95 \%$ CI \\
\hline Written health literacy & -4.23 & .18 & $-10.47,2.02$ \\
Family responsibility & -3.20 & .40 & $-10.73,4.32$ \\
Written health literacy X Family responsibility & 0.10 & .26 & $-0.07,0.27$ \\
Diabetes knowledge & -0.88 & .31 & $-2.58,0.83$ \\
& & & \\
Written health literacy & 2.61 & .14 & $-0.85,6.07$ \\
Family conflict & 3.52 & .10 & $-0.66,7.70$ \\
Written health literacy X Family conflict & -0.09 & .08 & $-0.18,0.01$ \\
Diabetes knowledge & -1.21 & .18 & $-3.00,0.58$ \\
& & & \\
Written health literacy & 0.50 & .50 & $-3.48,4.49$ \\
Parental supportive behavior & 1.11 & .70 & $-4.58,6.81$ \\
Written health literacy X Supportive behavior & -0.02 & .74 & $-0.16,0.11$ \\
Diabetes knowledge & -0.95 & .30 & $-2.80,0.89$ \\
& & & \\
Written health literacy & 3.14 & .09 & $-0.54,6.82$ \\
Parental non-supportive behavior & 7.82 & .06 & $-0.23,15.87$ \\
Written health literacy X Non-supportive behavior & -0.17 & .07 & $-0.36,0.01$ \\
Diabetes knowledge & -0.93 & .30 & $-2.71,0.84$ \\
\hline
\end{tabular}


Moderation Effect of Family Factors on the Relation between Diabetes Knowledge, Written Health Literacy, and Glycemic Control

\begin{tabular}{lccc}
\hline Predictor & $b$ & $p$ & $95 \%$ CI \\
\hline Written health literacy & -0.09 & .81 & $-0.86,0.67$ \\
Family responsibility & -0.01 & .98 & $-0.94,0.91$ \\
Written health literacy X Family responsibility & 0.00 & .92 & $-0.02,0.02$ \\
Diabetes knowledge & 0.05 & .62 & $-0.16,0.26$ \\
& & & \\
Written health literacy & -0.28 & .17 & $-0.68,0.12$ \\
Family conflict & -0.31 & .21 & $-0.79,0.17$ \\
Written health literacy X Family conflict & 0.01 & .19 & $0.00,0.02$ \\
Diabetes knowledge & 0.06 & .59 & $-0.15,0.26$ \\
& & & \\
Written health literacy & 0.07 & .75 & $-0.37,0.52$ \\
Parental supportive behavior & 0.12 & .70 & $-0.52,0.76$ \\
Written health literacy X Supportive behavior & 0.00 & .61 & $-0.02,0.01$ \\
Diabetes knowledge & 0.03 & .78 & $-0.18,0.24$ \\
& & & \\
Written health literacy & & .90 & $-0.43,0.42$ \\
Parental non-supportive behavior & 0.00 & .99 & $-0.80,1.06$ \\
Written health literacy X Non-supportive behavior & 0.00 & .87 & $-0.02,0.02$ \\
Diabetes knowledge & 0.06 & .57 & $0.15,0.26$ \\
\hline
\end{tabular}


Moderation Effect of Family Factors on the Relation between Diabetes Knowledge, Numeric Health Literacy, and Transition Readiness

\begin{tabular}{lccc}
\hline Predictor & $b$ & $p$ & $95 \%$ CI \\
\hline Numeric health literacy & -5.58 & .16 & $-13.36,2.20$ \\
Family responsibility & -1.02 & .38 & $-3.32,1.28$ \\
Numeric health literacy X Family responsibility & 0.18 & .09 & $-0.03,0.38$ \\
Diabetes knowledge & -1.81 & .03 & $-3.48,-0.14$ \\
& & & \\
Numeric health literacy & 1.88 & .38 & $-2.39,6.16$ \\
Family conflict & 0.11 & .87 & $-1.28,1.51$ \\
Numeric health literacy X Family conflict & -0.02 & .72 & $-0.15,0.11$ \\
Diabetes knowledge & -1.77 & .07 & $-3.67,0.12$ \\
& & & \\
Numeric health literacy & -0.90 & .75 & $-6.60,4.81$ \\
Parental supportive behavior & -0.61 & .54 & $-2.61,1.39$ \\
Numeric health literacy X Supportive behavior & 0.07 & .45 & $0.11,0.25$ \\
Diabetes knowledge & -1.71 & .06 & $-3.52,0.11$ \\
& & & \\
Numeric health literacy & 3.14 & .12 & $-0.86,7.13$ \\
Parental non-supportive behavior & 1.31 & .22 & $-0.81,3.42$ \\
Numeric health literacy X Non-supportive behavior & -0.10 & .28 & $-0.29,0.09$ \\
Diabetes knowledge & -1.67 & .07 & $-3.47,0.13$ \\
\hline
\end{tabular}


Moderation Effect of Family Factors on the Relation between Diabetes Knowledge, Numeric Health Literacy, and Glycemic Control

\begin{tabular}{lccc}
\hline Predictor & $b$ & $p$ & $95 \%$ CI \\
\hline Numeric health literacy & 0.02 & .96 & $-0.91,0.95$ \\
Family responsibility & 0.10 & .48 & $-0.18,0.37$ \\
Numeric health literacy X Family responsibility & -0.01 & .64 & $-0.03,0.02$ \\
Diabetes knowledge & 0.12 & .23 & $-0.08,0.32$ \\
& & & \\
Numeric health literacy & -0.22 & .37 & $-0.70,0.27$ \\
Family conflict & -0.01 & .93 & $-0.16,0.15$ \\
Numeric health literacy X Family conflict & 0.00 & .87 & $-0.01,0.02$ \\
Diabetes knowledge & 0.11 & .29 & $-0.10,0.33$ \\
& & & \\
Numeric health literacy & -0.04 & .91 & $-0.67,0.60$ \\
Parental supportive behavior & 0.01 & .93 & $-0.21,0.23$ \\
Numeric health literacy X Supportive behavior & 0.00 & .65 & $-0.02,0.02$ \\
Diabetes knowledge & 0.09 & .36 & $-0.11,0.29$ \\
& & & \\
Numeric health literacy & 0.23 & .28 & $-0.19,0.65$ \\
Parental non-supportive behavior & 0.31 & .01 & $0.09,0.53$ \\
Numeric health literacy X Non-supportive behavior & -0.02 & .08 & $-0.04,0.00$ \\
Diabetes knowledge & 0.15 & .13 & $-0.04,0.34$ \\
\hline
\end{tabular}

